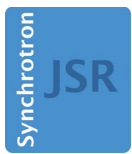

JOURNAL OF SYNCHROTRON RADIATION

ISSN 1600-5775

Received 14 April 2021

Accepted 13 July 2021

Edited by K. Kvashnina, ESRF - The European Synchrotron, France

Keywords: EXAFS oscillation; h.c.p. crystals; non-ideal axial ratio; Debye-Waller factor; anharmonic correlated Debye model.

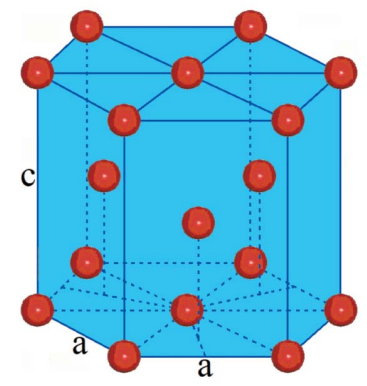

\section{Effect of the non-ideal axial ratio c/a on anharmonic EXAFS oscillation of h.c.p. crystals}

\author{
Tong Sy Tien* \\ University of Fire Prevention and Fighting, 243 Khuat Duy Tien, Hanoi 120602, Vietnam. \\ *Correspondence e-mail: tongsytien@yahoo.com
}

The temperature and wavenumber dependence of the extended X-ray absorption fine-structure (EXAFS) oscillation of hexagonal close-packed (h.c.p.) crystals have been calculated and analyzed under the effect of the non-ideal axial ratio $c / a$. The anharmonic EXAFS oscillation is presented in terms of the Debye-Waller factor using the cumulant expansion approach up to the fourth order. An effective calculation model is expanded and developed from the many-body perturbation approach and correlated Debye model using the anharmonic effective potential. This potential, depending on the non-ideal axial ratio $c / a$, is obtained from the first-shell near-neighbor contribution approach. A suitable analysis procedure is performed by evaluating the influence of EXAFS cumulants on the phase shift and amplitude reduction of the anharmonic EXAFS oscillation. The numerical results for crystalline zinc are found to be in good agreement with those obtained from experiments and other theoretical methods at various temperatures. The obtained results show that the present theoretical model is essential and effective in improving the accuracy for analyzing the experimental data of anharmonic EXAFS signals of h.c.p. crystals with a non-ideal axial ratio $c / a$.

\section{Introduction}

Extended X-ray absorption fine-structure (EXAFS) signals can be used to define the structural parameters and dynamic properties of many materials (Lytle et al., 1975; Beni \& Platzman, 1976; Greegor \& Lytle, 1979), so it has developed into a powerful technique and is the method of choice in many investigations in materials science (Yokoyama et al., 1996; Yokoyama, 1998; Lytle, 1999). However, thermal disorders in a crystal lattice cause anharmonicity that results in additional terms in the EXAFS oscillation (Eisenberger \& Brown, 1979; Lee et al., 1981; Tranquada \& Ingalls, 1983), leading to nonnegligible errors if these terms are ignored in structural parameters (Hung \& Rehr, 1997; Rehr \& Albers, 2000). Usually, the anharmonic corrections lead to revisions of the Gaussian distribution form of the Debye-Waller (DW) factor. This DW factor can be viewed as a result of averaging the single-scattering EXAFS formula over many near-neighbor pairs with a given radial pair distribution (RD) function (Tröger et al., 1994; Rehr \& Albers, 2000). The expanded DW factor is also described in detail using the moments of the $\mathrm{RD}$ function (or cumulants) in the ratio method (cumulant expansion approach) (Bunker, 1983; Crozier et al., 1988). This method consists of the separate analysis of phase and amplitude of the EXAFS signal at each temperature, taking a lowtemperature spectrum as reference (Bunker, 1983; Dalba et al., 1993). It is particularly suited to studying temperaturedependent variations of the first-shell parameters since it is mainly independent of theoretical inputs and allows a direct 
estimation of the quality of experimental data (Abd El All et al., 2013; Fornasini et al., 2001; Fornasini \& Grisenti, 2015). Usually, for disorders that are not too large, the ratio method can reproduce the asymmetric bond length distribution in the first coordination shell for many different materials (Sanson, 2010; Fornasini et al., 2017; Vila et al., 2018). And the first four cumulants can suffice to reproduce the complex bond-length distribution and have been confirmed for both crystalline and amorphous materials (Yokoyama et al., 1996, Yokoyama, 1998; Dalba et al., 1995, 1999; Fornasini \& Grisenti, 2015, Fornasini et al., 2017).

In many cases, the DW approximation is inadequate in disordered systems (Dalba \& Fornasini, 1997), and standard treatment of such vibrational and configurational disorder must often rely on a simpler model and phenomenological approximations (Dalba et al., 1998; Rehr \& Albers, 2000). In these typical approaches, an anharmonic correlated Einstein (ACE) model (Hung \& Rehr, 1997) is completed based on the correlated Einstein (CE) model (Sevillano et al., 1979) using the anharmonic effective (AE) potential. This AE potential (Hung \& Rehr, 1997) has taken into account the anharmonic effects and is based on the first-shell near-neighbor contribution (FSNNC) approach (Duc et al., 2018a). The ACE model can be performed based on the quantum statistical theory (Frenkel \& Rehr, 1993) (hereafter called the QACE model) (Hung \& Rehr, 1997) or the classical statistical theory (Stern et al., 1991) (hereafter called the CACE model) (Hung et al., 2014), which is successfully applied to investigate the anharmonic EXAFS cumulants of many various crystals (Hung \& Fornasini, 2007; Hung et al., 2008, 2014, 2017a,b, 2019; Tien et al., 2019; Duc, 2020; Tien, 2020a, 2021a). The QACE model has recently been developed based on the ACE model and the first-order perturbation by Tien (2020b). This QACE model has been successfully used to analyze the anharmonic EXAFS amplitude and phase of face-centered cubic crystals in a suitable procedure. Still, it has not yet been used to review the wavenumber dependence of the anharmonic EXAFS oscillation in detail. Moreover, the ACE models (QACE and CACE models) describe the atomic vibrations by the phonons having a unique correlated Einstein frequency, so they cannot mimic the acoustic phonon branches presenting in lattice crystals (Grimvall, 1999; Grosso \& Parravicini, 2000). Also, an anharmonic correlated Debye (ACD) model was completed based on the correlated Debye (CD) model (Beni \& Platzman, 1976) using the AE potential (Hung \& Rehr, 1997) and manybody perturbation (MBP) approach (Mahan, 1990) by Hung et al. (2010). This model can effectively treat the acoustic phonons branches present in lattice crystals because it describes the atomic vibrations by the phonons propagating with the speed of sound and having frequencies varying from 0 to the correlated Debye frequency. It was effectively used to investigate the anharmonic EXAFS spectra of body-centered cubic (b.c.c.) crystals (Hung et al., 2016) and face-centered cubic (f.c.c.) crystals (Duc et al., 2017, 2018b). Still, the ACD model has not yet been used for analyzing the anharmonic EXAFS oscillation and optimized for the expressions of cumulants, especially in the low-temperature limit.
In recent years, many metals of hexagonal close-packed (h.c.p.) structure have been widely used to make advanced materials in science and technology, such as beryllium (Be), cobalt (Co), cadmium (Cd), magnesium $(\mathrm{Mg})$, ruthenium $(\mathrm{Ru})$, zinc $(\mathrm{Zn})$, zirconium $(\mathrm{Zr})$, titanium (Ti) and yttrium $(\mathrm{Y})$ crystals (Vérité et al., 2007; Connétable et al., 2011; Podolskaya \& Krivtsov, 2012; Ghorai, 2018). These h.c.p. crystals have lower symmetry and isotropy than other cubic crystals such as f.c.c. crystals, b.c.c. crystals and diamond (DIA) crystals (Vérité et al., 2007; Podolskaya \& Krivtsov, 2012). The anharmonic EXAFS cumulants of h.c.p. crystals have also been obtained using the CACE model, QACE model (for the first three cumulants) and experimental EXAFS data by Hung et al. $(2008,2014,2017 b)$. However, the previous works only calculate approximately for h.c.p. crystals using an ideal axial ratio $c / a \simeq(8 / 3)^{1 / 2}$ (Hung et al., 2008, 2014, 2017b). This means that the effect of the non-ideal axial ratio cla on the anharmonic EXAFS oscillation has not been taken into account in the previous calculations. Therefore, the calculation and analysis of the effect of the non-ideal axial ratio $c / a$ on the anharmonic EXAFS oscillation of h.c.p. crystals using an extended ACD model and a suitable procedure will be a necessary addition to data analysis in the EXAFS technique.

The purpose of the present work is to expand and develop an effective model in calculating and analyzing the temperature and wavenumber dependence of the anharmonic EXAFS oscillation of h.c.p. crystals under the effect of the non-ideal axial ratio $c / a$. The primary objective is to demonstrate that the effect of the non-ideal axial ratio $c / a$ is significant and necessary to be considered in the anharmonic EXAFS oscillation analysis of h.c.p. crystals. In this work, the effect of the non-ideal axial ratio $c / a$ has been taken into account in the $\mathrm{AE}$ potential that depends on the structure parameters of h.c.p. crystals and obtained from the FSNNC approach, in which the Morse potential characterizes the interaction between a pair of atoms. An effective calculation-model is perfected based on the extended ACD model to calculate the anharmonic EXAFS cumulant. The anharmonic EXAFS oscillation is presented in terms of the DW factor using the cumulant expansion approach up to the fourth-order. A suitable analysis procedure is performed by evaluating the influence of cumulants on the phase shift and amplitude reduction to analyze the temperature and wavenumber dependence of the anharmonic EXAFS oscillation. Our numerical results for $\mathrm{Zn}$ are compared with those obtained from the ACE models and experiments (Hung et al., 2008, 2014, 2017b) at various temperatures. From these obtained results, we discuss the meaning and role of cumulants in analyzing the anharmonic EXAFS oscillation of h.c.p. crystals under the effect of the non-ideal axial ratio $c / a$, especially for high-order cumulants (third and fourth cumulants).

This article is organized as follows. The basic formulae of the anharmonic EXAFS oscillation and the calculation model are presented in Section 2. Section 3 calculates the effect of the non-ideal axial ratio $c / a$ on the AE potential and the first four anharmonic EXAFS cumulants of h.c.p. crystals using the extended ACD model. The numerical results in analyzing the 
anharmonic EXAFS oscillation for $\mathrm{Zn}$ are compared and discussed with experiments and other theoretical methods in Section 4. Section 5 gives the main conclusions about the present investigation.

\section{Basic formulae of the anharmonic EXADS oscillation}

The $K$-edge EXAFS oscillation includes non-Gaussian disorder for a polycrystalline material and is described within the framework of the single scattering and plane-wave approximations by (Tranquada \& Ingalls, 1983; Crozier et al., 1988; Tien, 2020b)

$\chi(k)=\frac{N S_{0}^{2}(k)}{k} F(k) \operatorname{Im}\left\{\left\langle\frac{\exp [-2 r / \lambda(k)]}{r^{2}} \exp (2 i k r)\right\rangle \exp [i \delta(k)]\right\}$

where $k$ is the photoelectron wavenumber, $S_{0}^{2}(k)$ is an amplitude reduction factor due to the many-body effect, $F(k)$ is the atomic backscattering amplitude, $N$ is the coordination number, the angular bracket $\langle\ldots\rangle$ is the average value in considering variations in bond lengths both due to thermal effects as well as static distortions, $\delta(k)$ is the net phase shift, $\lambda(k)$ is the electron effective mean free path, and $r$ is the instantaneous bond length between backscattering and absorbing atoms and depends on the temperature $T$.

The influence of temperature on the $K$-edge EXAFS oscillation of $\mathrm{Zn}$ is represented in Fig. 1. It can be seen that the thermal vibrations significantly influence the amplitude reduction and phase shift of the anharmonic EXAFS oscillation, as well as the decrease of the peak heights and their shifts to the left as the temperature $T$ increases, especially at higher temperatures.

Following the approach proposed by Freund et al. (1989), we expand the angular brackets $\left\langle\exp [-2 r / \lambda(k)] \exp (2 i k r) / r^{2}\right\rangle$ in equation (1) in a Taylor series about $R=\langle r\rangle$ to second-order and rewrite the thermal averages $\langle\exp (2 i k r)\rangle$ in terms of

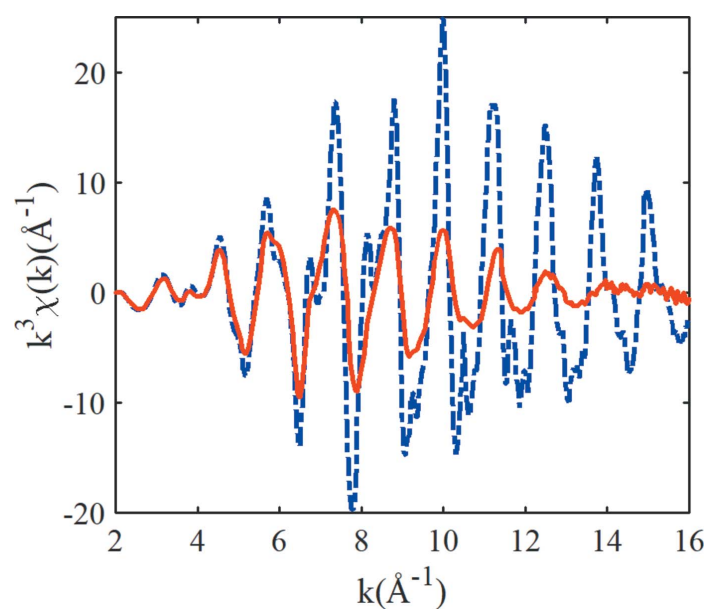

Figure 1

The $K$-edge EXAFS oscillations $k^{3} \chi(k)$ of $\mathrm{Zn}$ were obtained from the experimental EXAFS signals at $77 \mathrm{~K}$ (dashed-dotted blue line) and $300 \mathrm{~K}$ (solid red line) (Hung et al., 2008). anharmonic EXAFS cumulants using the cumulant expansion approach (Bunker, 1983; Crozier et al., 1988). Approximating to the fourth cumulant for small and moderate disorders and neglecting the small-term yields from the mean free path in the obtained expression, the $K$-edge EXAFS oscillation in the form $\chi(k, T)=A(k, T) \sin \Phi(k, T)$ is written as

$$
\begin{aligned}
\chi(k, T) \simeq & \frac{N S_{0}^{2}(k) \exp [-2 R / \lambda(k)]}{k R^{2}} \\
& \times F(k) \exp \left\{-2 k^{2} \sigma^{2}+\frac{2 k^{4}}{3} \sigma^{(4)}\right\} \\
& \times \sin \left\{2 k r_{0}+2 k \sigma^{(1)}-4 k \sigma^{2}\left[\frac{1}{R}+\frac{1}{\lambda(k)}\right]\right. \\
& \left.-\frac{4 k^{3}}{3} \sigma^{(3)}+\delta(k)\right\},
\end{aligned}
$$

where the coefficients $\sigma^{(n)}(T)$ are $n$ th-order cumulants and can be presented in terms of the power moments of the true RD function $\rho(T, r)$ (Fujikawa \& Miyanaga, 1993; Tröger et al., 1994), and $r_{0}$ is the equilibrium distance between the backscattering and absorbing atoms (Tranquada \& Ingalls, 1983; Yokoyama et al., 1989, 1996).

It can be seen that the amplitude $A(k, T)$ and phase $\Phi(k, T)$ of the anharmonic EXAFS oscillation depend on both the temperature $T$ and wavenumber $k$. From equation (2), the logarithm of the amplitude ratio $M\left(k, T_{1}, T_{2}\right)=$ $\ln \left[A\left(k, T_{2}\right) / A\left(k, T_{1}\right)\right]$ and the linear phase difference $\Delta \Phi\left(k, T_{1}, T_{2}\right)=\Phi\left(k, T_{2}\right)-\Phi\left(k, T_{1}\right)$ between temperatures $T_{2}$ and $T_{1}$ can be inferred in the form

$$
\begin{array}{rl}
M\left(k, T_{1}, T_{2}\right) \simeq & -2 k^{2}\left\{\sigma^{2}\left(T_{2}\right)-\sigma^{2}\left(T_{1}\right)\right\} \\
& +\frac{2 k^{4}}{3}\left\{\sigma^{(4)}\left(T_{2}\right)-\sigma^{(4)}\left(T_{1}\right)\right\}, \\
\Delta \Phi\left(k, T_{1}, T_{2}\right) \simeq 2 & k\left\{\sigma^{(1)}\left(T_{2}\right)-\sigma^{(1)}\left(T_{1}\right)\right\} \\
& -4 k\left\{\frac{1}{r_{0}}+\frac{1}{\lambda(k)}\right\}\left\{\sigma^{2}\left(T_{2}\right)-\sigma^{2}\left(T_{1}\right)\right\} \\
& -\frac{4 k^{3}}{3}\left\{\sigma^{(3)}\left(T_{2}\right)-\sigma^{(3)}\left(T_{1}\right)\right\},
\end{array}
$$

where the quantities $S_{0}^{2}(k), F(k), \lambda(k)$ and $\delta(k)$ in equation (2) are assumed to be the same at temperatures $T_{1}$ and $T_{2}$ (Tranquada \& Ingalls, 1983; Sanson, 2010; Fornasini et al., 2017), the contribution of the term $-2\left\{\left[R\left(T_{2}\right)-R\left(T_{1}\right)\right] / \lambda(k)+\right.$ $\left.\ln \left[R\left(T_{2}\right) / R\left(T_{1}\right)\right]\right\}$ is considered negligible in equation (3) (Dalba et al., 1993; Tröger et al., 1994; Tien, 2020b), and the approximate expression $1 / R(T) \simeq 1 / r_{0}$ is used in equation (4) (Crozier et al., 1988; Rehr \& Albers, 2000; Tien, 2020b).

Thus, the cumulants are critical for the quantitative treatment of the anharmonic EXAFS oscillation. The odd-order cumulants contribute primarily to the phase shift, and the even-order cumulants contribute to the amplitude reduction, as seen in equations (3) and (4). This means that the correct calculation of anharmonic EXAFS cumulants using a suitable model is essential to define the material structure accurately. 


\section{Calculation of the anharmonic EXAFS cumulants using the extended ACD model}

This section calculates the first four anharmonic EXAFS cumulants of h.c.p. crystals under the effect of the non-ideal axial ratio $c / a$ using the extended ACD model, where the AE potential depends on the structural parameter of h.c.p. crystals obtained from the FSNNC approach.

\subsection{AE potential within FSNNC approach}

In order to specify thermodynamic parameters of a material structure, it is necessary to determine the interatomic interaction potential and local force constants (Yokoyama et al., 1996; Hung \& Rehr, 1997). Let us consider an AE potential expanded up to the fourth-order around its minimum position as a function of the displacement of interatomic distance from equilibrium (Hung et al., 2008, 2014, 2017a; Tien et al., 2019). If ignoring the constant term, the AE potential is written as

$$
V_{\mathrm{eff}}(x)=\frac{1}{2} k_{0} x^{2}-k_{3} x^{3}+k_{4} x^{4}, \quad x=r-r_{0},
$$

where $x$ is the displacement, $k_{0}$ is the effective force constant, $k_{3}$ and $k_{4}$ are force constants describing the anharmonicity and bias in the RD function, and these local force constants are assumed to be temperature independent.

In relative vibrations of the backscattering and absorbing atoms, the AE potential (Hung \& Rehr, 1997) in the center of mass frame of the single bond pair of these atoms is given by

$$
V_{\mathrm{eff}}(x)=\varphi(x)+\sum_{i=1,2} \sum_{j \neq 1,2} \varphi\left(\varepsilon_{i} x \hat{R}^{0} \hat{R}_{i j}\right), \quad \varepsilon_{i}=\frac{\mu}{m_{i}},
$$

where $\mu=m_{1} m_{2} /\left(m_{1}+m_{2}\right)$ is the reduced mass of an atomic pair, $m_{i}$ is the mass of the $i$ th atom, sum $i$ is over absorber ( $i=1)$ and backscatterer $(i=2)$, sum $j$ is over their nearestneighbor atoms, $\hat{R}_{i j}$ is the unit vector along with bond between the $i$ th and $j$ th atoms, and $\hat{R}^{0}$ is the direction unit vector linking absorber and backscatterer.

In the model of h.c.p. crystal structure, each atom in the first shell is bonded to 12 other surrounding atoms, and the conventional unit cell is usually described by two lattice constants $a$ and $c$, as seen in Fig. 2. The ratio of the $c$-axis to $a$ axis is written as $e=c / a$, where $a$ is the basal plane edge length and $c$ is the height. This ratio characterizes the degree of

Figure 2

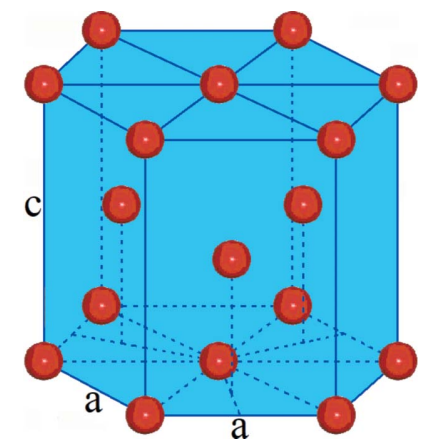

Model of the h.c.p. crystal structure. asymmetry and anisotropy of the crystal structure. For monatomic crystals, all atoms are similar with mass $m_{i}=m$, so the values of parameters $e_{1}, e_{2}, \varepsilon_{1}$ and $\varepsilon_{2}$ are calculated as $e_{1}=$ $e_{2}=e$ and $\varepsilon_{1}=\varepsilon_{2}=1 / 2$.

Considering the backscattering and absorbing atoms and their nearest-neighbor atoms to calculate the AE potential of this crystal structure from equation (6), we obtain the result as

$$
\begin{aligned}
V_{\mathrm{eff}}(x)= & \varphi(x)+4 \varphi(0)+2 \varphi\left(-\frac{1}{2} x\right)+4 \varphi\left(-\frac{1}{4} x\right)+4 \varphi\left(\frac{1}{4} x\right) \\
& +4 \varphi\left(-\frac{\sqrt{3}}{2\left(3 e^{2}+4\right)^{1 / 2}} x\right)+4 \varphi\left(\frac{\sqrt{3}}{2\left(3 e^{2}+4\right)^{1 / 2}} x\right) .
\end{aligned}
$$

The Morse potential (Morse, 1929; Girifalco \& Weizer, 1959) is often applied validly to determine the pair interaction potential of cubic metals, and it can be expressed in expanding around its minimum (position $x=0$ ) up to the fourth-order in the form as

$$
\varphi(x) \cong-D+D \alpha^{2} x^{2}-D \alpha^{3} x^{3}+7 D \alpha^{4} x^{4} / 12,
$$

where $\alpha$ describes the width of the pair interaction potential, and $D$ is the dissociation energy.

Using the Morse potential equation (8) to calculate the AE potential of h.c.p. crystals from equation (7) and if the overall constant in the result is ignored, the obtained result is

$$
\begin{aligned}
V_{\mathrm{eff}}(x)= & 2\left(\frac{3 e^{2}+7}{3 e^{2}+4}\right) D \alpha^{2} x^{2}-\frac{5}{4} D \alpha^{3} x^{3} \\
& +\frac{7\left(333 e^{4}+888 e^{2}+736\right)}{384\left(3 e^{2}+4\right)^{2}} D \alpha^{4} x^{4}
\end{aligned}
$$

The local force constants $k_{0}, k_{3}$ and $k_{4}$ of h.c.p. crystals are deduced from comparing equation (5) with equation (9) as follows,

$$
\begin{gathered}
k_{0}=4\left(\frac{3 e^{2}+7}{3 e^{2}+4}\right) D \alpha^{2}, \quad k_{3}=\frac{5}{4} D \alpha^{3}, \\
k_{4}=\frac{7\left(333 e^{4}+888 e^{2}+736\right)}{384\left(3 e^{2}+4\right)^{2}} D \alpha^{4} .
\end{gathered}
$$

Note that the contribution of the h.c.p. crystal structure with non-ideal axial ratio $c / a$ to the AE potential in equation (9) and the local force constants in equation (10) are obtained from the FSNNC approach (Duc et al., 2018a). In this approach, the influence of nearest surrounding atoms on the thermal vibrations of backscattering and absorbing atoms is determined by the projection of their pair interaction along the bonding direction (Negele \& Orland, 1972). Moreover, the calculated expressions of the AE potential and local force constants of the h.c.p. crystals are similar to that of the f.c.c. crystal if the h.c.p. crystals have an ideal axial ratio $c / a=(8 / 3)^{1 / 2}$.

Thus, the effect of the non-ideal axial ratio $c / a$ on thermodynamic parameters of h.c.p. crystals is described via the dependence of the AE potential and local force constants on the structural parameter $e$, as seen in equations (9) and (10). The present calculation model took into account three- 
dimensional interactions, even though it only uses a simple approach with the one-dimensional model.

\subsection{Correlated Debye frequency and temperature}

The ACD model is derived from the dualism of an elementary particle in quantum theory and is perfected based on the CD model (Beni \& Platzman, 1976) using the AE potential (Hung \& Rehr, 1997) and MBP approach (Mahan, 1990). In this model, the atomic vibrations can be quantized and treated as phonons, and the anharmonicity is the result of the phonon-phonon interactions (Beni \& Platzman, 1976; Feynman, 1998). Therefore, the atomic vibrations of the crystal lattice can be described as a system consisting of many phonons, each of which corresponds to a wave that has frequency $\omega(q)$ with wavenumber $q$ varying in the first Brillouin zone (Kittel, 2004; Hung et al., 2010; Duc et al., 2018a, Tien, 2021b),

$$
\begin{aligned}
\omega(q) & =2\left(\frac{k_{0}}{m}\right)^{1 / 2}\left|\sin \left(\frac{q a}{2}\right)\right| \\
& =4 \alpha\left[\frac{\left(3 e^{2}+7\right) D}{\left(3 e^{2}+4\right) m}\right]^{1 / 2}\left|\sin \left(\frac{q a}{2}\right)\right|, \quad|q| \leq \frac{\pi}{a}
\end{aligned}
$$

where $q$ is the phonon wavenumber, $a$ is the lattice constant in one-dimensional systems, and the effective force constant $k_{0}$ is given by equation (10).

The wavenumber dependence of the frequency of h.c.p. crystals is represented in Fig. 3. It can be shown that the frequency is maximum at the bounds of the first Brillouin zone of the linear chain with the values of the wavenumber as $q= \pm \pi / a$ (Hung et al., 2016). Hence, the correlated Debye frequency $\omega_{\mathrm{D}}$ and temperature $\theta_{\mathrm{D}}$ are derived from equation (20) as follows,

$$
\begin{aligned}
& \omega_{\mathrm{D}}=4 \alpha\left[\frac{\left(3 e^{2}+7\right) D}{\left(3 e^{2}+4\right) m}\right]^{1 / 2}, \\
& \theta_{\mathrm{D}}=\frac{\hbar \omega_{\mathrm{D}}}{k_{\mathrm{B}}}=\frac{4 \hbar \alpha}{k_{\mathrm{B}}}\left[\frac{\left(3 e^{2}+7\right) D}{\left(3 e^{2}+4\right) m}\right]^{1 / 2},
\end{aligned}
$$

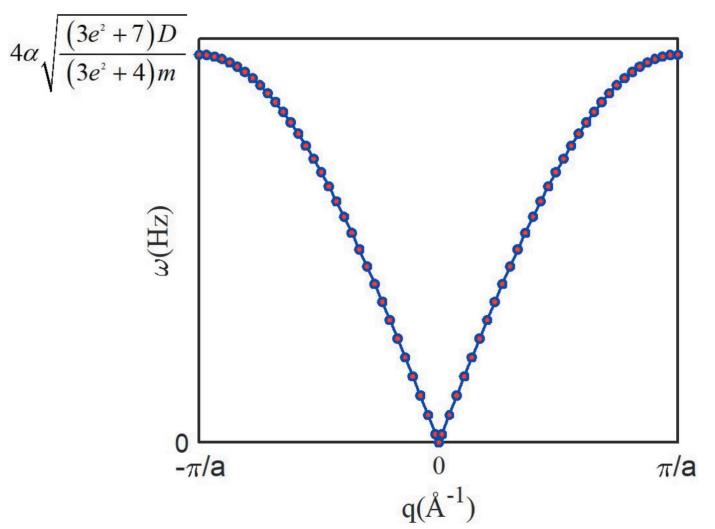

Figure 3

Wavenumber dependence of the frequency of h.c.p. crystals in the ACD model. where $\omega_{\mathrm{D}}$ can be treated using the formula $\omega_{\mathrm{D}}=c q_{\mathrm{D}}, k B$ is the Boltzmann constant and $h$ is the reduced Planck constant.

Thus, the thermal vibrations of atoms are described by $N$ phonons that propagate with the sound speed $c$ and the frequencies varying from 0 to the correlated Debye temperature frequency $\omega_{\mathrm{D}}$ in the crystal lattice having volume $V$. The effect of the non-ideal axial ratio $c / a$ on the correlated Debye temperature $\theta_{\mathrm{D}}$ and frequency $\omega_{\mathrm{D}}$ of h.c.p. crystals can be described via the structural parameter $e$, as seen in equation (12).

\subsection{Anharmonic EXAFS cumulants within the extended ACD model}

The Hamiltonian includes the anharmonic effects of a system written as a summation of the harmonic and anharmonic components (Yokoyama et al., 1996; Feynman, 1998; Hung et al., 2010),

$$
H=H_{0}+H_{\mathrm{a}}
$$

where

$$
H_{0}=-\frac{\hbar^{2}}{2 \mu} \frac{d^{2}}{d x^{2}}+\frac{1}{2} k_{0} x^{2}
$$

is the harmonic Hamiltonian term and $H_{\mathrm{a}}=-k_{3} x^{3}+k_{4} x^{4}$ is the anharmonic Hamiltonian term.

In the ACD model, the displacement $x$ of the $n$th atom is related to the displacement operator $A_{q}$ of phonons and can be expressed via the displacement $u_{n}$ of a one-dimensional chain (Horner, 1974; Miyanaga \& Fujikawa, 1994; Hung et al., 2016; Duc et al., 2018a, Tien, 2021b),

$$
x=u_{n+1}-u_{n}=\sum_{q} \exp (\text { iqan }) f(q) A_{q},
$$

where the function $f(q)$ and displacement operator $A_{q}$ are defined with the following properties,

$$
\begin{array}{r}
f(q)=\left[\frac{\hbar}{2 N m \omega(q)}\right]^{1 / 2}[\exp (\text { iqa })-1], \quad A_{q}=A_{-q}^{+}, \\
{\left[A_{q}, A_{q^{\prime}}\right]=0 .}
\end{array}
$$

Then the moments $\left\langle x^{k}\right\rangle$ can be calculated with the aid of the MBP approach (Mahan, 1990) and are given by

$$
\left\langle x^{k}\right\rangle=\frac{\sum_{q_{1}, q_{2}, \ldots, q_{k}} f\left(q_{1}\right) f\left(q_{2}\right) \ldots f\left(q_{k}\right)\left\langle A_{q_{1}} A_{q_{2}} \ldots A_{q k} S(\beta)\right\rangle_{0}}{\langle S(\beta)\rangle_{0}},
$$

where the function $S(\beta)$ is written via the anharmonic Hamiltonian $\hat{H}_{\mathrm{a}}(\tau)$ in an interactive representation as

$$
\begin{aligned}
S(\beta) & =\sum_{n=0}^{\infty} \frac{(-1)^{n}}{n !} \int_{0}^{\beta} \mathrm{d} \tau_{1} \ldots \int_{0}^{\beta} \mathrm{d} \tau_{1} T\left[\hat{H}_{\mathrm{a}}\left(\tau_{1}\right) \ldots \hat{H}_{\mathrm{a}}\left(\tau_{n}\right)\right], \\
\hat{H}_{\mathrm{a}}(\tau) & =\exp \left(\tau H_{0}\right) H_{\mathrm{a}} \exp \left(-\tau H_{0}\right), \quad \beta=1 / k_{\mathrm{B}} T .
\end{aligned}
$$

Following the approach proposed by Hung et al. (2010, 2016), using the Wick theorem for the T-product in the integral for the harmonic phonon Green function and properties of the 
phonon displacement operator $A_{q}$, we calculate the moments $\left\langle x^{k}\right\rangle$ according to the procedures depicted in equations (13)(17). After using the local force constants $k_{0}, k_{3}$ and $k_{4}$ of h.c.p. crystals given from equation (10) and converting from variable $q$ to variable $p$ by the formula

$$
p=q a / 2,
$$

we calculate the temperature dependence of the first four EXAFS cumulants and obtain the following results.

The first cumulant describes the net thermal expansion (NTE) or the lattice disorder (Crozier et al., 1988; Tröger et al., 1994) in the form

$$
\begin{aligned}
\sigma^{(1)}(T) & =\langle r\rangle-r_{0}=\langle x\rangle \\
& =\frac{15 \hbar}{64 \pi D \alpha}\left(\frac{3 e^{2}+4}{3 e^{2}+7}\right)^{2} \int_{0}^{\pi / 2} \mathrm{~d} p \omega(p) \frac{1+Z(p)}{1-Z(p)} .
\end{aligned}
$$

The second cumulant describes the parallel mean-square relative displacement (MSRD) $\sigma^{2}$ that relates to the projected vibrational density of states (VDOS) $\rho(\omega)$ (Crozier et al., 1988; Tröger et al., 1994) in the form

$$
\begin{aligned}
\sigma^{2}(T) & \equiv \sigma^{2}=\left\langle(r-R)^{2}\right\rangle=\left\langle x^{2}\right\rangle-\langle x\rangle^{2} \\
& =\frac{\hbar}{4 \pi D \alpha^{2}}\left(\frac{3 e^{2}+4}{3 e^{2}+7}\right) \int_{0}^{\pi / 2} \mathrm{~d} p \omega(p) \frac{1+Z(p)}{1-Z(p)} .
\end{aligned}
$$

The third cumulant is the mean cubic relative displacement (MCRD) and describes the asymmetry of the RD function (Crozier et al., 1988; Dalba et al., 1995, 1998) in the form

$$
\begin{aligned}
\sigma^{(3)}(T)= & \left\langle(r-R)^{3}\right\rangle=\left\langle(x-\langle x\rangle)^{3}\right\rangle=\frac{5 \hbar^{2}}{64 \pi^{2} D^{2} \alpha^{3}}\left(\frac{3 e^{2}+4}{3 e^{2}+7}\right)^{3} \\
& \times \int_{0}^{\pi / 2} \mathrm{~d} p_{1} \int_{-\pi / 2}^{\pi / 2-p_{1}} \mathrm{~d} p_{2} \frac{\omega\left(p_{1}\right) \omega\left(p_{2}\right) \omega\left(p_{1}+p_{2}\right)}{\omega\left(p_{1}\right)+\omega\left(p_{2}\right)+\omega\left(p_{1}+p_{2}\right)} \\
& \times\left\{1+6 \frac{\omega\left(p_{1}\right)+\omega\left(p_{2}\right)}{\omega\left(p_{1}\right)+\omega\left(p_{2}\right)-\omega\left(p_{1}+p_{2}\right)}\right. \\
& \left.\times \frac{Z\left(p_{1}\right) Z\left(p_{2}\right)-Z\left(p_{1}+p_{2}\right)}{\left[Z\left(p_{1}\right)-1\right]\left[Z\left(p_{2}\right)-1\right]\left[Z\left(p_{1}+p_{2}\right)-1\right]}\right\} .
\end{aligned}
$$

The fourth cumulant characterizes the anharmonic contribution to the EXAFS amplitude and describes the flatness of the RD function from a harmonic Gaussian shape (Crozier et al., 1988; Dalba et al., 1995, 1998, 1999) in the form

$$
\begin{aligned}
& \sigma^{(4)}(T)=\left\langle(r-R)^{4}\right\rangle-3\left(\sigma^{2}\right)^{2}=\left\langle(x-\langle x\rangle)^{4}\right\rangle-3\left(\sigma^{2}\right)^{2} \\
& \simeq \frac{21 \hbar^{3}}{\pi^{3} D^{3} \alpha^{4}} \frac{\left(333 e^{4}+888 e^{2}+736\right)\left(3 e^{2}+4\right)^{2}}{16384\left(3 e^{2}+7\right)^{4}} \\
& \times \int_{0}^{\pi / 2} \mathrm{~d} p_{1} \times \int_{0}^{\pi / 2-p_{1}} \mathrm{~d} p_{2} \\
& \times \int_{-\pi / 2}^{\pi / 2-\left(p_{1}+p_{2}\right)} \mathrm{d} p_{3} \frac{\omega\left(p_{1}\right) \omega\left(p_{2}\right) \omega\left(p_{3}\right) \omega\left(p_{1}+p_{2}+p_{3}\right)}{\omega\left(p_{1}\right)+\omega\left(p_{2}\right)+\omega\left(p_{3}\right)+\omega\left(p_{1}+p_{2}+p_{3}\right)}\{1+ \\
& 8 \frac{Z\left(p_{1}\right) Z\left(p_{2}\right) Z\left(p_{3}\right)-Z\left(p_{1}+p_{2}+p_{3}\right)}{\left[Z\left(p_{1}\right)-1\right]\left[Z\left(p_{2}\right)-1\right]\left[Z\left(p_{3}\right)-1\right]\left[Z\left(p_{1}+p_{2}+p_{3}\right)-1\right]} \\
& \times \frac{\omega\left(p_{1}\right)+\omega\left(p_{2}\right)+\omega\left(p_{3}\right)}{\omega\left(p_{1}\right)+\omega\left(p_{2}\right)+\omega\left(p_{3}\right)-\omega\left(p_{1}+p_{2}+p_{3}\right)} \\
& +6 \frac{Z\left(p_{1}\right) Z\left(p_{2}\right)-Z\left(p_{3}\right) Z\left(p_{1}+p_{2}+p_{3}\right)}{\left[Z\left(p_{1}\right)-1\right]\left[Z\left(p_{2}\right)-1\right]\left[Z\left(p_{3}\right)-1\right]\left[Z\left(p_{1}+p_{2}+p_{3}\right)-1\right]} \\
& \left.\times \frac{\omega\left(p_{3}\right)+\omega\left(p_{1}+p_{2}+p_{3}\right)}{\omega\left(p_{1}\right)+\omega\left(p_{2}\right)-\omega\left(p_{3}\right)-\omega\left(p_{1}+p_{2}+p_{3}\right)}\right\},
\end{aligned}
$$

where the frequency $\omega(p)$ is given from equation (11), and the function $Z(p)$ is identified as $Z(p)=\exp \{\beta \hbar \omega(p)\}$ with $p=q a / 2$.

To calculate the anharmonic EXAFS cumulants in the hightemperature (HT) and low-temperature (LT) limits and at the zero point (ZP) from equations (19)-(22) based on the ACD model, we use the approximations $Z(p) \approx 1+\beta \hbar \omega(p)$ in the HT limit $(T \rightarrow \infty), 1 / Z^{n}(p) \approx 0$ with $n>1$ in the LT limit $(T \rightarrow 0)$, and $1 / Z^{n}(p) \approx 0$ with $n>0$ at the $\mathrm{ZP}(T=0)$.

The obtained expressions using the present ACD model in the LT and HT limits and at the ZP of the first four EXAFS cumulants of h.c.p. crystals taking into account the effect of non-ideal axial ratio $c / a$ are given in Fig. 4. These expressions describe the anharmonicity contribution of thermal vibrations to the EXAFS oscillation in the HT limit and the influence of quantum effects on the EXAFS oscillation in the LT limit. Also, they describe the contribution of zero-point energy to the EXAFS oscillation at the ZP. In the case of h.c.p. crystals with ideal axial ratio $c / a=(8 / 3)^{1 / 2}$, our obtained expressions of the first four EXAFS cumulants in the HT limit are $\sigma^{(1)}=$ $3 k_{\mathrm{B}} T / 20 D \alpha, \sigma^{(2)}=k_{\mathrm{B}} T / 5 D \alpha^{2}, \sigma^{(3)}=3\left(k_{\mathrm{B}} T\right)^{2} / 50 D^{2} \alpha^{3}$ and $\sigma^{(4)}=133\left(k_{\mathrm{B}} T\right)^{3} / 5000 D^{3} \alpha^{4}$. It can be seen that the first three cumulants $\sigma^{(1)}, \sigma^{(2)}$ and $\sigma^{(3)}$ are similar to those calculated from the obtained expressions of the QACE (Hung et al., 2008, 2017b) and CACE (Hung et al., 2014) models, and the fourth cumulant $\sigma^{(4)}$ is slightly smaller than the result $\sigma^{(4)}=$ $137\left(k_{\mathrm{B}} T\right)^{3} / 5000 D^{3} \alpha^{4}$ obtained from the CACE model (Hung et al., 2014). Our obtained expressions of the first four EXAFS cumulants at the $\mathrm{ZP}$ are $\sigma^{(1)} \simeq 3 \hbar / 2 \pi(5 D m)^{1 / 2}, \sigma^{(2)} \simeq$ $2 \hbar / \pi \alpha(5 D m)^{1 / 2}, \quad \sigma^{(3)} \simeq 88 \hbar^{2} / 375 \pi^{2} \alpha D m$, and $\sigma^{(4)} \simeq$ $1976 \hbar^{3} / 1875 \sqrt{5} \pi^{3} \alpha m^{3 / 2} D^{3 / 2}$. It can be seen that the first three cumulants $\sigma^{(1)}, \sigma^{(2)}$ and $\sigma^{(3)}$ are smaller than the results $\sigma^{(1)} \simeq$ $3 \hbar / 4(10 D m)^{1 / 2}, \sigma^{(2)} \simeq \hbar / \alpha(10 D m)^{1 / 2}$ and $\sigma^{(3)} \simeq \hbar^{2} / 20 \alpha D m$, respectively, calculated from the obtained expressions of the QACE model (Hung et al., 2008, 2017b). Meanwhile, the first four cumulants $\sigma^{(1)}, \sigma^{(2)}, \sigma^{(3)}$ and $\sigma^{(4)}$ obtained from the 


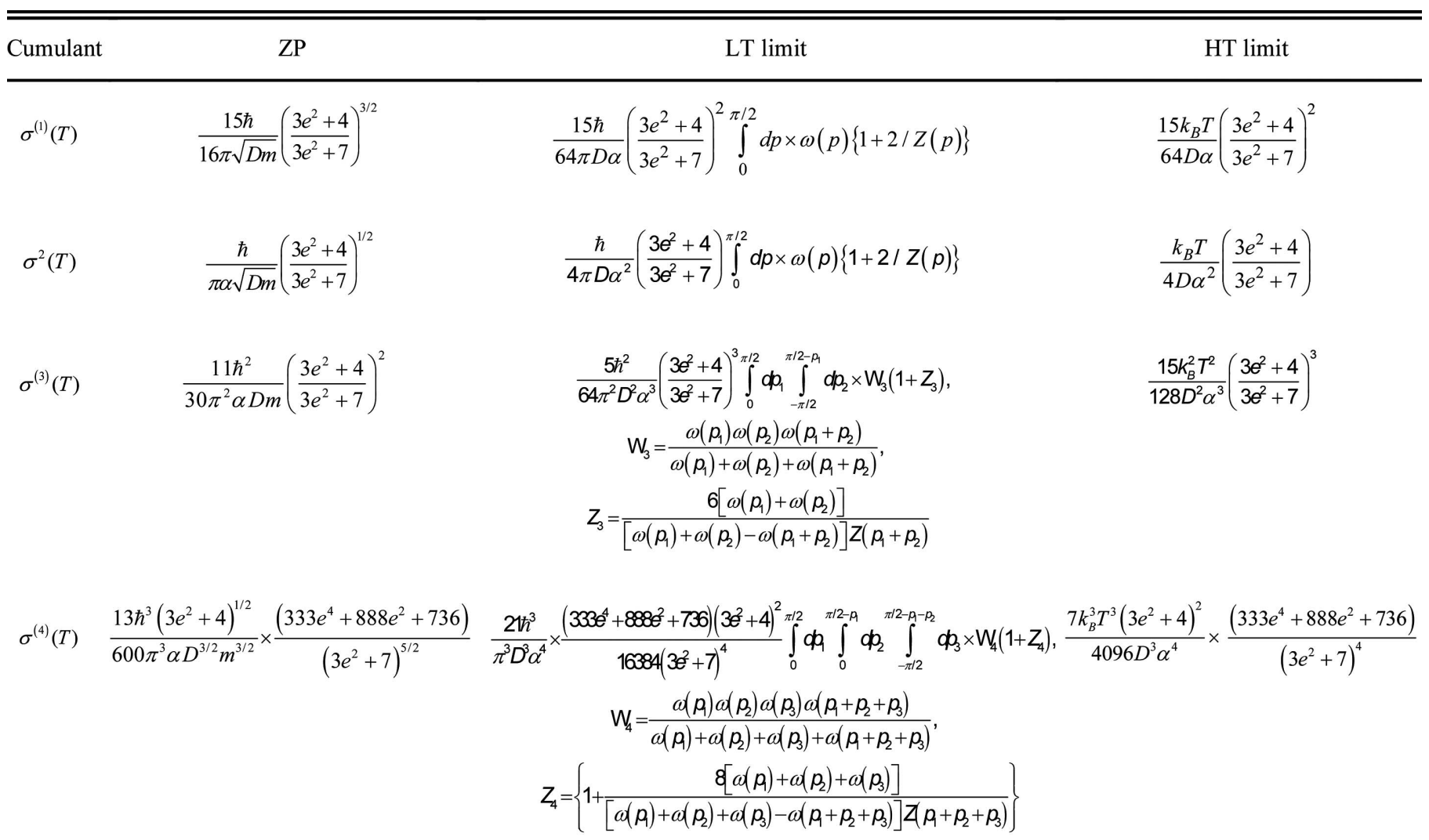

Figure 4

The dependence on the structural parameter $e$ of the first four anharmonic EXAFS cumulants of h.c.p. crystals in the LT and HT limits and at the ZP obtained using the present ACD model.

CACE model (Hung et al., 2014) progress gradually to zero because this model cannot calculate quantum effects. The small difference between the present ACD and QACE (Hung et al., 2008, 2017b) models in the obtained results is because the present ACD model took into account the dispersion relation of thermal vibrations, while the QACE model (Hung et al., 2008, 2017b) only uses an effective frequency to describe these thermal vibrations.

Note that the present ACD model has taken into account the effect of the non-ideal axial ratio $c / a$ on the EXAFS oscillation of h.c.p. crystals. In contrast, the ACE models (Hung et al., 2008, 2014, 2017b) do not consider this effect because they only use an approximation of the ideal axial ratio $c / a \approx(8 / 3)^{1 / 2}$. Moreover, as can be seen in equations (19)(22), using the present ACD model with converting variable $p=q a / 2$ to calculate the anharmonic EXAFS cumulants, we have obtained expressions independent of the lattice constant $a$ in one-dimensional systems. Also, we have calculated expressions of the first four cumulants at the ZP in explicit forms and optimized expressions of the third and fourth cumulants in the LT limit, as seen in Fig. 4. Meanwhile, other ACD models in the previous works (Hung et al., 2010, 2016; Duc et al., 2017, 2018b) have not yet performed calculations for these expressions.
Thus, a calculation model of the anharmonic EXAFS cumulants of h.c.p. crystals has been perfected based on the extended ACD model (hereafter cited as the EACD model). The effect of the non-ideal axial ratio $c / a$ on the first four anharmonic EXAFS cumulants is described via their dependence on the structural parameter $e$. The obtained temperature-dependent expressions using the present EACD model of the first four EXAFS cumulants can satisfy all their fundamental properties. These expressions have described both the influences of anharmonic effects at high temperatures on the classical limit and that of the quantum effects at low temperatures on the zero-point energy.

\section{Numerical results and discussions}

In order to discuss the effectiveness and development of the present theoretical model for calculating and analyzing the effect of the non-ideal axial ratio $c / a$ on the temperature and wavenumber dependence of the anharmonic EXAFS oscillation of h.c.p. crystals in this work, we use the formulae in Section 2 and the obtained expressions in Section 3 to calculate numerical results for $\mathrm{Zn}$. Firstly, we use the physical parameters of $\mathrm{Zn}$ to calculate the local force constants, the correlated Debye temperature and frequency, and the first 
four EXAFS cumulants in the temperature range from $0 \mathrm{~K}$ to $700 \mathrm{~K}$. Then, we analyze the anharmonic EXAFS oscillation via the phase differences and the logarithm of amplitude ratios in the temperature range from $100 \mathrm{~K}$ to $700 \mathrm{~K}$ with reference values at $100 \mathrm{~K}$ and in the wavenumber range from $0 \AA^{-1}$ to $20 \AA^{-1}$. Our results are obtained using the EACD model in both cases with and without considering the effect of the nonideal axial ratio $c / a$. These results are compared with each other as well as with the corresponding results obtained from the QACE and CACE models by Hung et al. (2008, 2014, $2017 b)$. Moreover, our numerical results are also compared with experimental data measured at the Hamburg Synchrotron Radiation Laboratory (HASYLAB), German Electron Synchrotron (DESY) (Hamburg, Germany) and beamline 8 (BL8), Synchrotron Light (SLRI) (Nakhon Ratchasima, Thailand) by Hung et al. (2014, 2017b). In these comparisons, the obtained results using the ACE models (Hung et al., 2008, $2014,2017 b$ ) are in the temperature range from $0 \mathrm{~K}$ to $700 \mathrm{~K}$, the measured values at HASYLAB (Hung et al., 2014) are at $77 \mathrm{~K}$ and $300 \mathrm{~K}$, and the measured values at SLRI (Hung et al., $2017 b$ ) are at $300 \mathrm{~K}, 400 \mathrm{~K}, 500 \mathrm{~K}$ and $600 \mathrm{~K}$. Herein, the error values of measurements at SLRI have been determined from re-analysis of original EXAFS data provided by Hung et al., and they have also not been published in previous articles. Lastly, we evaluate the advancement of the present theoretical model in calculating and analyzing the anharmonic EXAFS oscillation of h.c.p. crystals under the effect of the non-ideal axial ratio $c / a$.

In calculations of $\mathrm{Zn}$, we use the atomic mass $m=65.3771$ (Marinenko \& Foley, 1975), lattice constants $a=$ $2.6594 \AA, c=4.9368 \AA$ and $e=1.8563$ (Jette \& Foote, 1935), and Morse potential parameters $D=0.1698 \mathrm{eV}, \alpha=$ $1.7054 \AA^{-1}$ and $r_{0}=2.7931 \AA$ (Hung et al., 2008). Using the EACD model and taking into account the effect of the non-ideal axial ratio $c / a=1.8563$ to calculate the thermodynamic parameters of $\mathrm{Zn}$ from equations (10) and (12), we obtain the local force constants $k_{0}=2.3887 \mathrm{eV} \AA^{-2}, k_{3}=1.0528 \mathrm{eV} \AA^{-3}$ and $k_{4}=0.9871 \mathrm{eV}^{-4}$, and the correlated Debye frequency $\omega_{\mathrm{D}}=$ $3.7552 \times 10^{13} \mathrm{~Hz}$ and temperature $\theta_{\mathrm{D}}=$ $286.8415 \mathrm{~K}$. It can be seen that our obtained results have a clear difference in comparison with the corresponding results obtained from ACE models with approximating the ideal axial ratio $c / a \simeq$ $(8 / 3)^{1 / 2}$ (Hung et al., 2008, 2014). Moreover, in comparison with experimental values (Hung et al., 2008, 2014), our obtained results also agree better than those obtained from these ACE models.

Figure 5
This means that the effect of the non-ideal axial ratio c/a should still be taken into account in the calculations of the thermodynamic parameters of h.c.p. crystals, especially when the deviation of the axial ratio $c / a$ compared with $(8 / 3)^{1 / 2}$ is large.

The temperature dependences of the $(a)$ first cumulant $\sigma^{(1)}(T),(b)$ second cumulant $\sigma^{2}(T),(c)$ third cumulant $\sigma^{(3)}(T)$ and $(d)$ fourth cumulant $\sigma^{(4)}(T)$ of $\mathrm{Zn}$ are shown in Fig. 5. Our obtained results using the EACD model are calculated by equations (19)-(22) in both cases with and without taking into account the effect of the non-ideal axial ratio $c / a$. The results taking into account the effect of the non-ideal axial ratio $c / a$ on the anharmonic EXAFS oscillation are calculated using the value $e=1.8563$. Meanwhile, the obtained results without considering this effect are approximately calculated using the value $e \simeq(8 / 3)^{1 / 2}$ as the ideal axial ratio $c / a$. As can be seen in Fig. 5, our results agree well with those obtained from the CACE model within the LT range and experiment (Hung et al., 2014), and the QACE model and another experiment (Hung et $a l ., 2017 b$ ) for the first three cumulants, in which the ACE models (Hung et al., 2014, 2017b) do not consider the effect of the non-ideal axial ratio cla. Also, in comparison with experimental values (Hung et al., 2017b), the obtained results
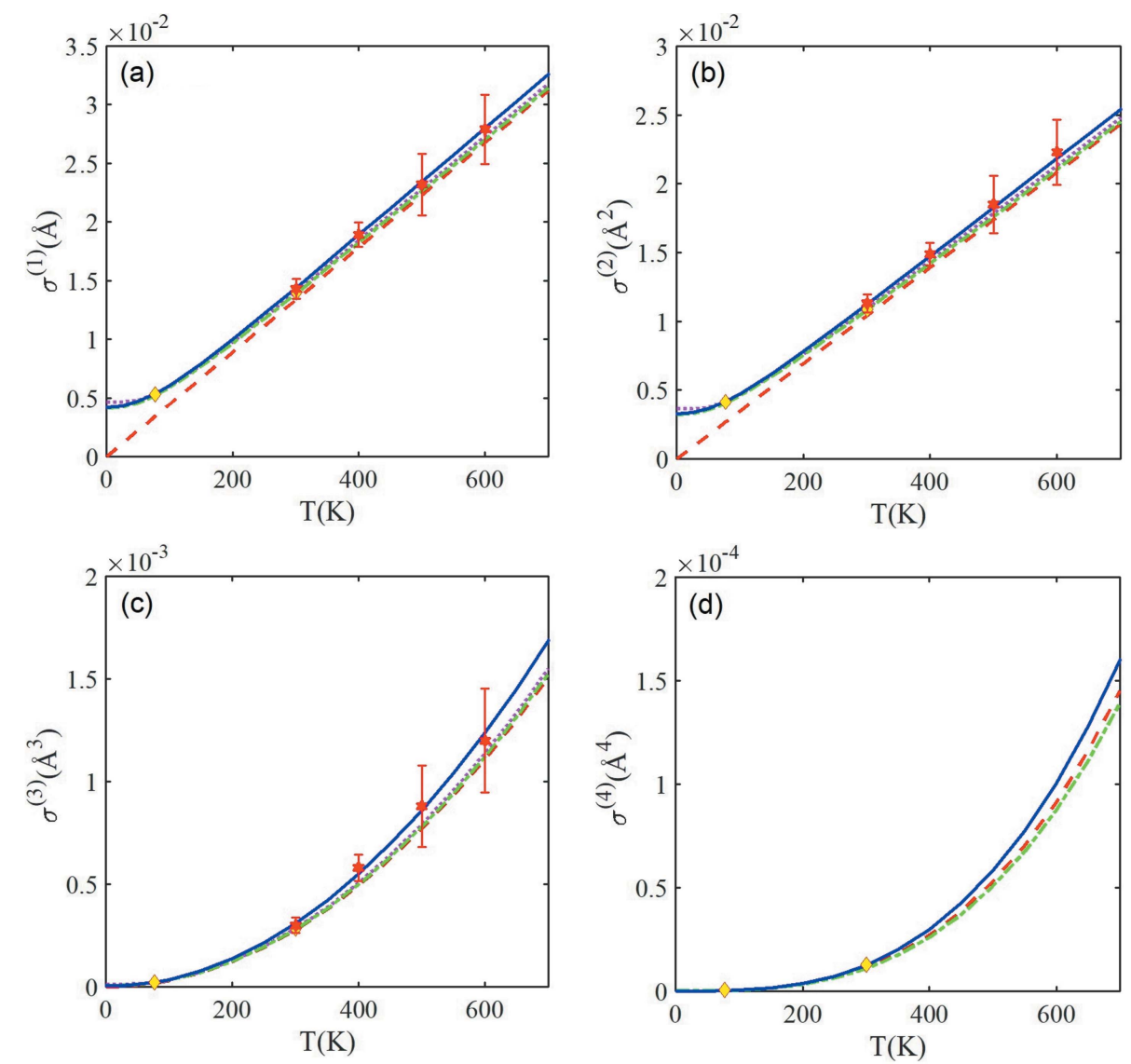

Temperature dependence of the $(a)$ first, $(b)$ second, $(c)$ third and $(d)$ fourth cumulants of $\mathrm{Zn}$ obtained using the EACD model with non-ideal (solid blue lines) and ideal (dashed-dotted green lines) axial ratios $c / a$, the CACE model (dashed red lines) and experiment (full yellow diamonds) (Hung et al., 2014), and the QACE (dotted magenta lines) model and another experiment (full red hexagrams with red error bars) (Hung et al., 2017b). 
Table 1

The first four anharmonic EXAFS cumulants of Zn obtained using the EACD model with non-ideal and ideal axial ratios $c / a$, the QACE and CACE models, and experiments.

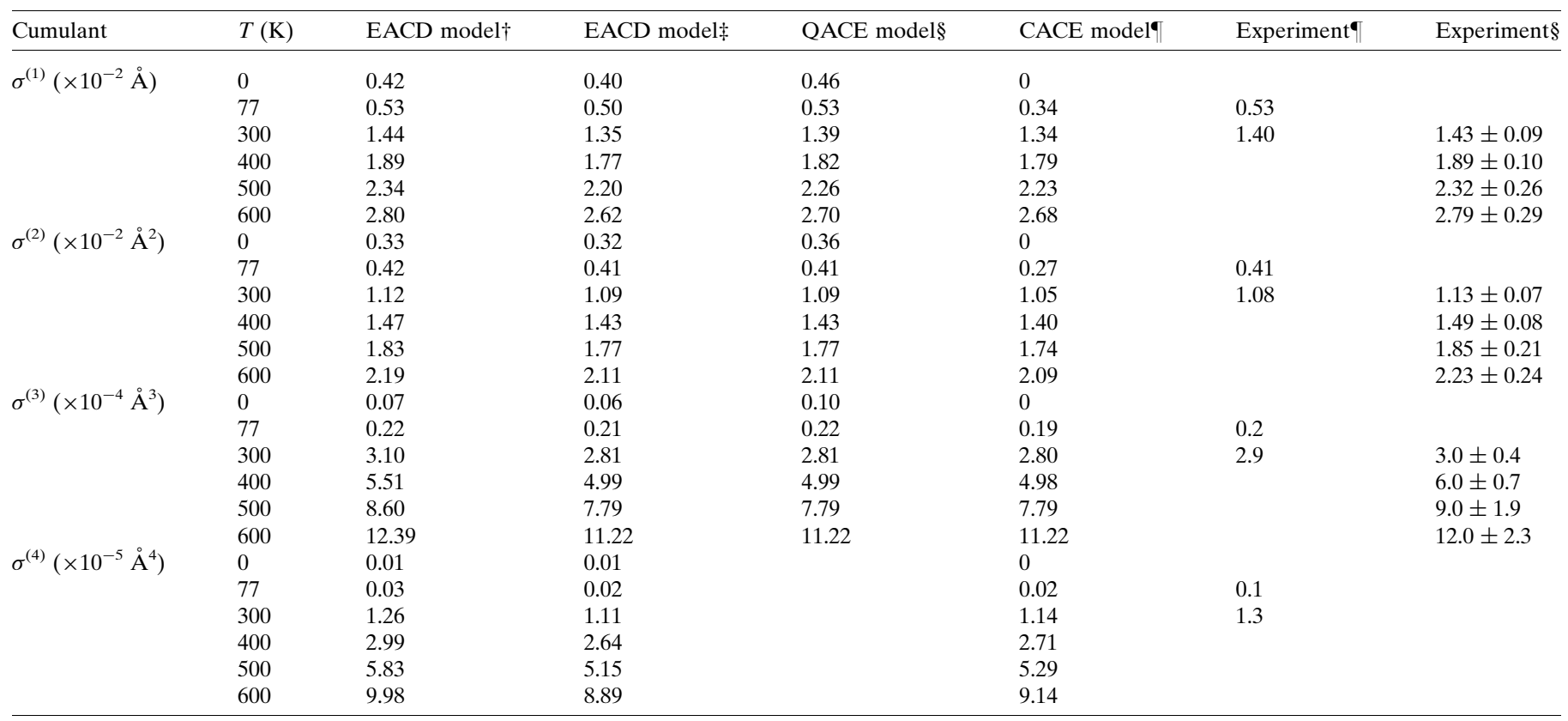

$\dagger$ Calculation with the non-ideal axial ratio $c / a$ in this work. $\ddagger$ Calculation with the ideal axial ratio $c / a$ in this work. $\S$ Hung et al. (2017b). $\uparrow$ Hung et al. (2014).

using the EACD model taking into account the effect of the non-ideal axial ratio $c / a$ agree better than those obtained from other models, as seen in Fig. 5.

The values of the first four EXAFS cumulants of $\mathrm{Zn}$ at $0 \mathrm{~K}$, $77 \mathrm{~K}, 300 \mathrm{~K}, 400 \mathrm{~K}, 500 \mathrm{~K}, 600 \mathrm{~K}$ and $700 \mathrm{~K}$ are given in Table 1, in which our results are calculated using the EACD model with non-ideal and ideal axial ratios $c / a$. The suitable agreement of our values in comparison with those obtained from the CACE model (Hung et al., 2014) within the LT range, the QACE model (Hung et al., 2017b) and experiments (Hung et al., 2014, 2017b) can be clearly seen. Still, there is a significant difference between the obtained values with the nonideal axial ratio $c / a$ and those obtained with the ideal axial ratio $c / a$. The effect of the non-ideal axial ratio $c / a$ clearly increases the values of low-order cumulants (first and second cumulants) and significantly increases the value of high-order cumulants, especially at high temperatures. Moreover, the obtained values with the non-ideal axial ratio $c / a$ are a better agreement with experimental values (Hung et al., 2014, 2017b) than those obtained with the ideal axial ratio $c / a$, especially compared with experimental values obtained by Hung et al. $(2017 b)$, as seen in Table 1.

Herein, there is a limitation in comparing the experimental values measured at HASYLAB and SLRI by Hung et al., as seen in Fig. 5 and Table 1. The measured values at HASYLAB (Hung et al., 2014) were published without error values, and we also have not received the original data from Hung et al. in order to redefine these values. Meanwhile, the error values of measurements at SLRI (Hung et al., 2017b) have been determined from re-analysis of the original EXAFS data provided by Hung et al., but these error values are quite large at $500 \mathrm{~K}$ and $600 \mathrm{~K}$, especially for the third EXAFS cumulant (uncertainties are about $21 \%$ and $19 \%$ ). The characterization of the magnitude of these errors can also be found in some experimental results for other materials (Stern et al., 1991; Dalba et al., 1993, 1999; Yokoyama, 1998; Fornasini \& Grisenti, 2015).

Comparisons with the above experimental values can influence the evaluated results on the suitability of results obtained from different methods if their difference is not great enough, as in this study. However, for evaluating the behavior of theoretical calculation models, comparisons based mainly on the average values of the experiment can still obtain the necessary scientific values (Yokoyama et al., 1989; Dalba et al., 1990; Strauch et al., 1996; Hung \& Rehr, 1997; Hung \& Fornasini, 2007; Beccara \& Fornasini, 2008; Fornasini et al., 2017). This is because the average value characterizes the probability of obtaining results close to this value for the plurality of the measurements taken (Xia et al., 2000; Lira \& Wöger, 2001).

Therefore, the obtained evaluations for the present calculation model in comparison with other methods are valid, although there are still certain limitations in using the above experimental values in this work.

The wavenumber dependence of $(a)$ the logarithm of amplitude ratios $M(k, T)=\ln [A(k, T) / A(k, 100 K)]$ and $(b)$ the phase differences $\Delta \Phi(k, T)=\Phi(k, T)-\Phi(k, 100 K)$ of $\mathrm{Zn}$ at $T=300 \mathrm{~K}$ and $T=500 \mathrm{~K}$ are shown in Fig. 6. Our obtained results using the EACD model are calculated using equations (3) and (4) in both cases with and without taking into account the effect of the non-ideal axial ratio $c / a$, in which the anharmonic EXAFS cumulants are given by equations 
(19)-(22). The effect of the non-ideal axial ratio $c / a$ on the anharmonic EXAFS oscillation is described by comparing the results obtained with the non-ideal axial ratio $c / a$ with those obtained with the ideal axial ratio $c / a$. The comparison results show that the effect of the non-ideal axial ratio $c / a$ on the logarithm of amplitude ratio and the phase difference is significant at high temperatures and large wavenumbers, especially for the phase difference, as seen in Fig. 6.

The values of the logarithm of the amplitude ratios $M(k, T)$ and the phase differences $\Delta \Phi(k, T)$ of $\mathrm{Zn}$ are given in Table 2. Our results are calculated using the EACD model with non-ideal and ideal axial ratios $c / a$ at $T=300 \mathrm{~K}$ and $500 \mathrm{~K}$ with $k=10 \AA^{-1}$ and $20 \AA^{-1}$. It can be clearly seen that the values of $M(k, T)$ and $\Delta \Phi(k, T)$ decrease with increasing temperature $T$ and decrease rapidly with increasing wave-
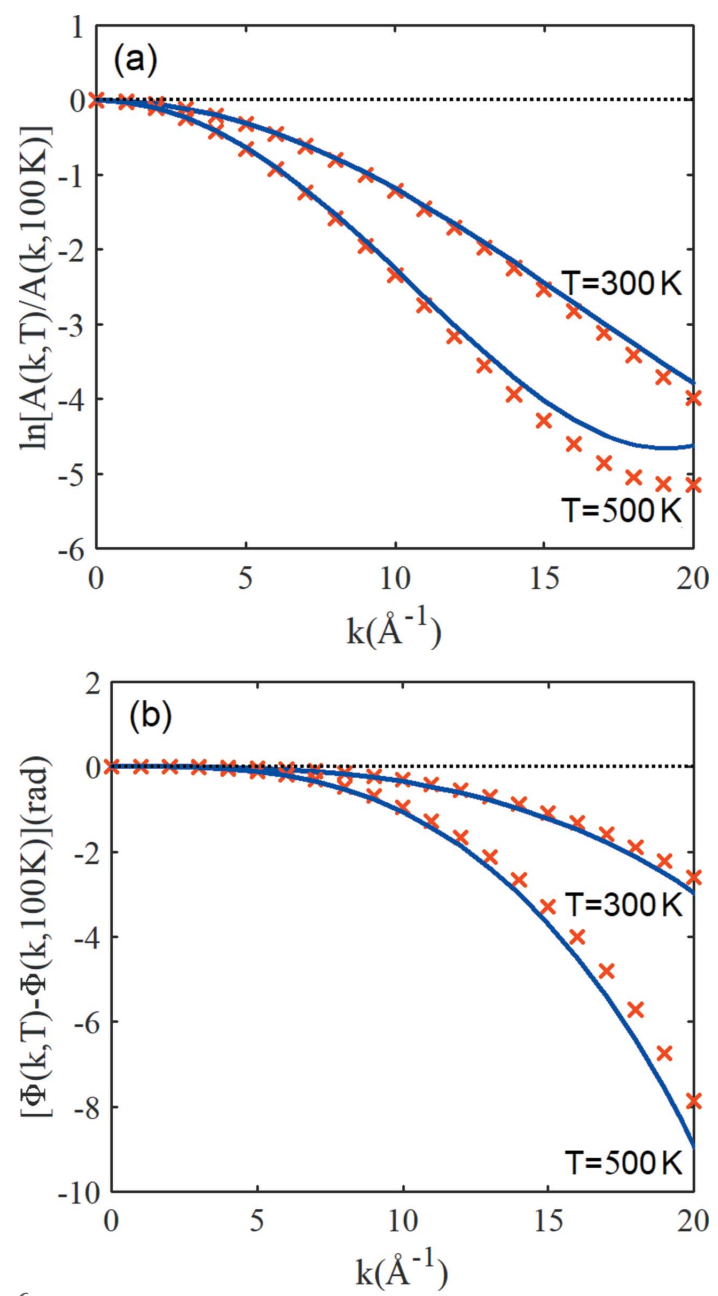

Figure 6

Wavenumber dependence of $(a)$ the logarithm of amplitude ratios and (b) the phase differences of $\mathrm{Zn}$ with non-ideal (solid blue lines) and ideal (red crosses signs) axial ratios $c / a$ obtained using the EACD model at $300 \mathrm{~K}$ and $500 \mathrm{~K}$ with reference values at $100 \mathrm{~K}$.
The logarithm of amplitude ratios $M(k, T)$ and the phase differences $\Delta \Phi(k, T)$ of $\mathrm{Zn}$ with non-ideal and ideal axial ratios $c / a$ are calculated using the EACD model.

\begin{tabular}{lllllll}
\hline Quantity & $T(\mathrm{~K})$ & $k\left(\AA^{-1}\right)$ & $\begin{array}{l}\text { Ideal axial } \\
\text { ratio } c / a\end{array}$ & $\begin{array}{l}\text { Non-ideal } \\
\text { axial ratio } c / a\end{array}$ & $\begin{array}{l}\text { Value } \\
\text { change }\end{array}$ & $\begin{array}{l}\text { Percentage } \\
\text { change }\end{array}$ \\
\hline \multirow{2}{*}{$M(k, T)$} & 300 & 10 & -1.22 & -1.18 & +0.04 & -3.28 \\
& 300 & 15 & -2.54 & -2.43 & +0.11 & -4.33 \\
& 300 & 20 & -4.01 & -3.78 & +0.23 & -5.74 \\
& 500 & 10 & -2.35 & -2.24 & +0.11 & -4.68 \\
& 500 & 15 & -4.29 & -4.02 & +0.27 & -6.29 \\
$\Delta \Phi(k, T)$ & 500 & 20 & -5.15 & -4.54 & +0.61 & -11.84 \\
& 300 & 10 & -0.31 & -0.34 & -0.03 & +9.68 \\
& 300 & 15 & -1.07 & -1.18 & -0.11 & +10.28 \\
& 300 & 20 & -2.60 & -2.91 & -0.10 & +11.92 \\
& 500 & 10 & -0.95 & -1.05 & -0.37 & +11.25 \\
& 500 & 15 & -3.29 & -3.66 & -0.98 & +12.47 \\
\hline
\end{tabular}

number $k$. Also, the effect of the non-ideal axial ratio $c / a$ significantly increases the value of $M(k, T)$ and clearly reduces the value of $\Delta \Phi(k, T)$ with increasing temperature $T$ and wavenumber $k$. However, the effect of the non-ideal axial ratio cla on the values of $M(k, T)$ is smaller and changes faster than this effect on the values of $\Delta \Phi(k, T)$, as seen in Table 2 .

Thus, the effect of the non-ideal axial ratio $c / a$ is significant and is necessarily taken into account in analyzing the anharmonic EXAFS oscillation of h.c.p. crystals, especially at high temperatures and large wavenumbers. This effect is stronger and the deviation of the ratio cla compared with $(8 / 3)^{1 / 2}$ is larger, which also increases the logarithm of the amplitude ratio and reduces the phase difference of the anharmonic EXAFS oscillation. This means that the effect of the non-ideal axial ratio $c / a$ clearly reduces the EXAFS amplitude reduction and significantly increases the EXAFS phase shift, especially with the axial ratio $c / a$ being much different from $(8 / 3)^{1 / 2}$.

The temperature and wavenumber dependence of $(a)$ the logarithm of the amplitude ratio $M(k, T)$ and $(b)$ the phase difference $\Delta \Phi(k, T)$ with their component terms of $\mathrm{Zn}$ are shown in Fig. 7. These surfaces are presented to visually describe the role and variation with increasing temperature and wavenumber of the components containing the cumulants that contribute to the amplitude reduction and phase shift of the anharmonic EXAFS oscillation. Our results are obtained using the EACD model taking into account the effect of the non-ideal axial ratio $c / a$. Herein, $M_{1}(k, T)$ and $M_{2}(k, T)$ are denoted as contributions to $M(k, T)$ of terms containing the second and fourth cumulants in equation (3), respectively. Likewise, $\Delta \Phi_{1}(k, T), \Delta \Phi_{2}(k, T)$ and $\Delta \Phi_{3}(k, T)$ are denoted as contributions to $\Delta \Phi(k, T)$ of terms containing the first, second and third cumulants in equation (4), respectively. As can be seen in Fig. 7(a), both $M_{1}(k, T)$ and $M_{2}(k, T)$ contribute primarily to the result of $M(k, T)$, in which the variation with temperature and wavenumber of $M_{2}(k, T)$ is faster than that of $M_{1}(k, T)$. Still, $M_{1}(k, T)$ reduces the value of $M(k, T)$, and $M_{2}(k, T)$ increases the value of $M(k, T)$. Also, the contribution of $\Delta \Phi_{3}(k, T)$ to the result of $\Delta \Phi(k, T)$ is the largest even though the contributions of $\Delta \Phi_{1}(k, T)$ and $\Delta \Phi_{2}(k, T)$ to the result of $\Delta \Phi(k, T)$ are significant. This is because the contribution of $\Delta \Phi_{1}(k, T)$ to the value of $\Delta \Phi(k, T)$ is an opposite 
Table 3

The logarithm of the amplitude ratios $M(k, T)$, the phase differences $\Delta \Phi(k, T)$ and their component terms of $\mathrm{Zn}$ are calculated using the EACD model with the non-ideal axial ratio $c / a$.

\begin{tabular}{|c|c|c|c|c|c|c|c|c|c|c|c|c|}
\hline$T(\mathrm{~K})$ & $\begin{array}{l}k \\
\left(\AA^{-1}\right)\end{array}$ & $M_{1}$ & $M_{2}$ & $M$ & $\begin{array}{l}M_{2} / M_{1} \\
(\%)\end{array}$ & $\begin{array}{l}\Delta \Phi_{1} \\
(\mathrm{rad})\end{array}$ & $\begin{array}{l}\Delta \Phi_{2} \\
(\mathrm{rad})\end{array}$ & $\begin{array}{l}\Delta \Phi_{3} \\
(\mathrm{rad})\end{array}$ & $\begin{array}{l}\Delta \Phi \\
(\mathrm{rad})\end{array}$ & $\begin{array}{l}\Delta \Phi_{1} / \Delta \Phi \\
(\%)\end{array}$ & $\begin{array}{l}\Delta \Phi_{2} / \Delta \Phi \\
(\%)\end{array}$ & $\begin{array}{l}\Delta \Phi_{3} / \Delta \Phi \\
(\%)\end{array}$ \\
\hline 200 & 10 & -0.62 & 0.02 & -0.60 & -3.23 & 0.08 & -0.06 & -0.14 & -0.12 & -66.67 & 40.00 & 116.67 \\
\hline 200 & 15 & -1.39 & 0.11 & -1.28 & -8.59 & 0.12 & -0.09 & -0.47 & -0.44 & -27.28 & 20.45 & 106.81 \\
\hline 200 & 20 & -2.47 & 0.35 & -2.12 & -14.17 & 0.16 & -0.13 & -1.09 & -1.06 & -15.09 & 12.26 & 102.83 \\
\hline 400 & 10 & -2.00 & 0.19 & -1.81 & -9.50 & 0.26 & -0.21 & -0.69 & -0.64 & -40.63 & 32.81 & 107.82 \\
\hline 400 & 15 & -4.51 & 0.98 & -3.53 & -27.76 & 0.39 & -0.32 & -2.35 & -2.28 & -17.11 & 14.04 & 103.07 \\
\hline 400 & 20 & -8.00 & 3.13 & -4.87 & -39.13 & 0.51 & -0.42 & -5.49 & -5.40 & -9.44 & 7.78 & 101.67 \\
\hline 600 & 10 & -3.42 & 0.67 & -2.75 & -19.59 & 0.44 & -0.36 & -1.64 & -1.56 & -28.21 & 23.08 & 105.13 \\
\hline 600 & 15 & -7.70 & 3.31 & -4.49 & & 0.66 & -0.54 & -5.41 & -5.29 & -12.48 & 10.21 & 102.27 \\
\hline 600 & 20 & -13.69 & 10.69 & -3.00 & -78.89 & 0.88 & -0.72 & -13.15 & -12.99 & -6.77 & 5.54 & 101.23 \\
\hline
\end{tabular}

sign and not much larger than that of $\Delta \Phi_{2}(k, T)$ to the value of $\Delta \Phi(k, T)$, as seen in Fig. $7(b)$.

Thus, the contribution of $M_{1}(k, T)$ to the value of $M(k, T)$ and the contributions of $\Delta \Phi_{1}(k, T), \Delta \Phi_{2}(k, T)$ and $\Delta \Phi_{3}(k, T)$ to the value of $\Delta \Phi(k, T)$ of $\mathrm{Zn}$ (h.c.p. crystal) are similar to the corresponding contributions of Mo (b.c.c. crystal) (Tien, 2020a), Cu and Ni (f.c.c. crystal) (Tien, 2020b, Tien, 2021a) and Ge (diamond crystal) (Tien, 2021b). However, the contribu-

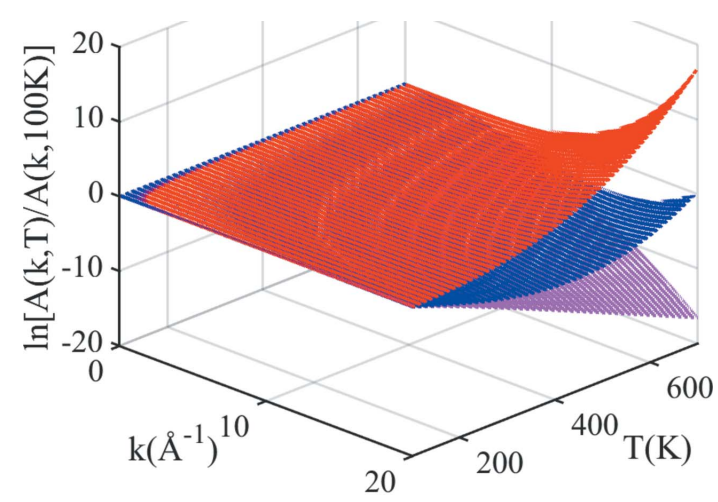

(a)

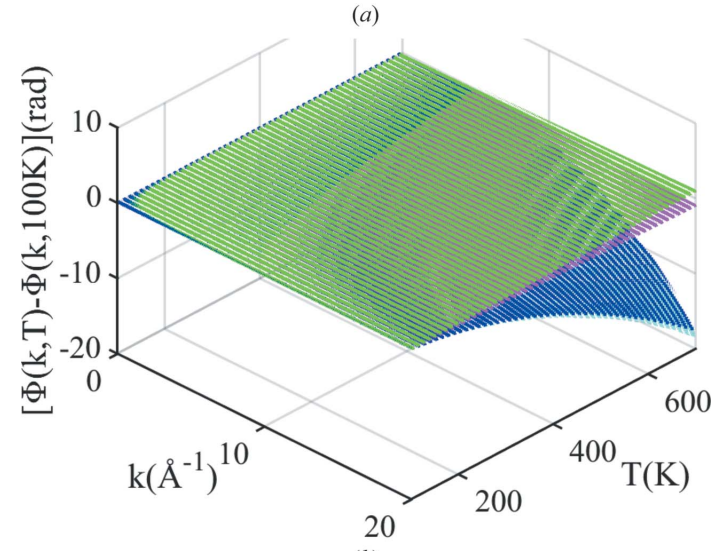

(b)

Figure 7

Temperature and wavenumber dependence of $(a)$ the logarithm of the amplitude ratio $M(k, T)$ and $(b)$ the phase difference $\Delta \Phi(k, T)$ with their component terms of $\mathrm{Zn}$ obtained using the EACD model with the nonideal axial ratio $c / a$ in the following cases: calculating all of the terms in equations (3) or (4) (blue mesh-surfaces) and calculating only the term containing the first cumulant in equation (4) (green mesh-surface), the second cumulant in equations (3) or (4) (magenta mesh-surfaces), the third cumulant in equation (4) (cyan mesh-surface), and the fourth cumulant in equation (3) (red mesh-surface). tion of $M_{2}(k, T)$ to the value of $M(k, T)$ of $\mathrm{Zn}$ (h.c.p. crystal) is significantly stronger than this corresponding contribution of Mo (b.c.c. crystal) (Tien, 2020a), $\mathrm{Cu}$ and $\mathrm{Ni}$ (f.c.c. crystal) (Tien, 2020b, 2021a), and Ge (diamond crystal) (Tien, 2021b).

The values of the logarithm of the amplitude ratios $M(k, T)$ and the phase differences $\Delta \Phi(k, T)$, and their component terms of $\mathrm{Zn}$ are given in Table 3. Our results are calculated using the EACD model with non-ideal axial ratio $c / a$ at $T=$ $200 \mathrm{~K}, 400 \mathrm{~K}$ and $600 \mathrm{~K}$ with $k=10 \AA^{-1}$ and $20 \AA^{-1}$. It can be clearly seen that the ratio $M_{2}(k, T) / M_{1}(k, T)$ is negative, and its absolute value increases sharply to a sizable one with fastincreasing temperature $T$ and increasing wavenumber $k$, in which $M_{1}(k, T)$ has the same sign as $M(k, T)$ and $M_{2}(k, T)$ has the opposite sign as $M(k, T)$. Meanwhile, the absolute values of the ratios $\Delta \Phi_{1}(k, T) / \Phi(k, T)$ and $\Delta \Phi_{2}(k, T) / \Phi(k, T)$ gradually decrease to approximately the same value with fastincreasing temperature $T$ and increasing wavenumber $k$, in which the ratio $\Delta \Phi_{1}(k, T) / \Phi(k, T)$ is negative and the ratio $\Delta \Phi_{2}(k, T) / \Phi(k, T)$ is positive. This results in the value of the ratio $\Delta \Phi_{3}(k, T) / \Phi(k, T)$ gradually progressing to $100 \%$ with fast-increasing temperature $T$ and increasing wavenumber $k$, as seen in Table 3.

Thus, the term $M_{2}(k, T)$ increases the value and contributes significantly to calculating the logarithm of the amplitude ratio $M(k, T)$, and the term $\Delta \Phi_{3}(k, T)$ reduces the value and contributes mainly to calculating the phase difference $\Delta \Phi(k, T)$. The influences of $M_{2}(k, T)$ on $M(k, T)$ and $\Delta \Phi_{3}(k, T)$ on $\Delta \Phi(k, T)$ are stronger at the higher temperatures and larger wavenumbers. The influence caused by the wavenumber is stronger than that caused by the temperature. This means that the fourth cumulant has a strong influence on the amplitude reduction, and the third cumulant has the greatest influence on the phase shift, especially at high temperatures and large wavenumbers. This result is essential and useful to analyze the anharmonic EXAFS oscillation from experimental data.

Currently, using the FEFFIT program suite and other theoretical fitting standards in the experimental XAFS data analysis technique is being performed and developed in many laboratories worldwide using properly established processes (Vila et al., 2018; Kas et al., 2020; Ravel \& Newville, 2020). In these processes, a non-linear best fit to the experimental XAFS data by a suitable theoretical code is capable of 
extracting reliable information of the structural parameters and dynamic properties of materials (Newville, 2001; Bunker, 2010). For EXAFS spectra, single scattering is dominant because the main contribution to the anharmonic EXAFS oscillation is given by the first shell (Rennert \& Hung, 1988; Stern et al., 1991). This is why, in theoretical fitting standards, only the $K$-edge EXAFS oscillation of the first shell is used to analyze measurement results of the experimental EXAFS signals (Bunker, 2010; Ravel \& Newville, 2020).

The IFEFFIT program suite (Newville, 2001; Newville \& Ravel, 2020) has many outstanding features and is designed to fit experimental EXAFS data to theoretical calculations from the well known FEFF code (Rehr et al., 1991; Kas et al., 2020) that yields atomic scattering amplitudes and phase shifts used in many EXAFS fitting models, as well as various other properties (Zabinsky et al., 1995; Ankudinov et al., 1998). This program suite enables extracting data from experimental data, Fourier transforms, Fourier filter and fitting the data to reference data from experiment or theory (Bunker, 2010). The fitting can be done in $k$ or $R$ space, with a wide range of options given for modeling EXAFS data (Jorissen \& Rehr, 2013). This includes a full suite of tools and methods for EXAFS analysis, including functions for pre-edge removal and normalization of EXAFS data, background removal using the Autobk method, and fitting EXAFS oscillation with theoretical calculations for scattering amplitudes and phase shifts (Newville \& Ravel, 2020).

Usually, the scattering path formalism and cumulant expansion from the $F E F F$ code are used as the basic building blocks of EXAFS data analysis to give a flexible and robust parameterization of most problems (Newville, 2001), in which these theoretical scattering factors and anharmonic EXAFS cumulants are provided to refine the fitting model and the extracted information from experimental data of Ravel \& Newville (2005). However, the reliability of the obtained results depends on the accuracy of the theoretical calculations and physical approximations implemented in the FEFF code (Fornasini \& Grisenti, 2015).

The wavenumber dependence of the $K$-edge EXAFS oscillation $k^{2} \chi(k)$ of $\mathrm{Zn}$ at $300 \mathrm{~K}$ is shown in Fig. 8. Our obtained results using the EACD model taking into account the effect of the non-ideal axial ratio $c / a$ is derived from modifying equation (2) in the FEFF code for the anharmonic EXAFS cumulants given by equations (19)-(22). It can be seen that our results agree with those obtained from the QACE model and experiment (Hung et al., 2017b) and another experiment (Hung et al., 2008), in which the QACE model does not consider the effect of the non-ideal axial ratio $c / a$ and the fourth EXAFS cumulant. Also, in comparison with experimental EXAFS signals (Hung et al., 2008, 2017b), the obtained results using the EACD model taking into account the effect of the non-ideal axial ratio $c / a$ agree better than those obtained from the QACE models, especially for the large wavenumber range and in comparison with experimental EXAFS signals obtained by Hung et al. (2017b), as seen in the peak heights and their shifts of the anharmonic EXAFS signals obtained from experiments and other theoretical

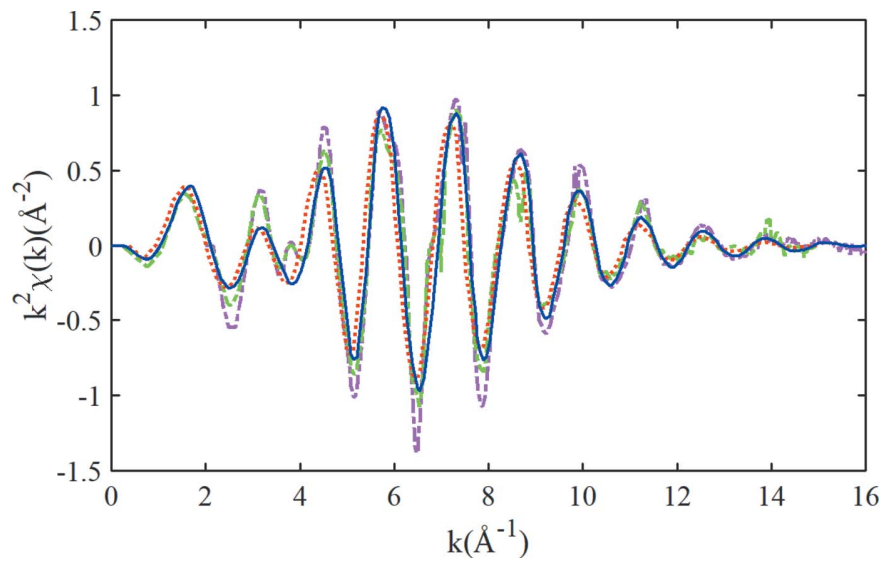

Figure 8

The $K$-edge EXAFS oscillation $k^{2} \chi(k)$ of $\mathrm{Zn}$ at $300 \mathrm{~K}$ obtained using the EACD model taking into account the effect of the non-ideal axial ratio cla (solid blue lines), the QACE model (dotted red lines) and experimental EXAFS signals (dashed-dotted green lines) (Hung et al., 2017b), and other experimental EXAFS signals (dashed-dotted magenta lines) (Hung et al., 2008).

models in Fig. 8. This is because the fourth EXAFS cumulant increases the amplitude, as seen in equation (2), and the effect of the non-ideal axial ratio $c / a$ tends to reduce the amplitude reduction and increase the phase differences of the anharmonic EXAFS oscillation, as seen from the evaluation results in Fig. 6 and Table 2. The obtained results also show that the ratio method is able to reproduce the $K$-edge EXAFS signal in the first coordination shell of $\mathrm{Zn}$ using the IFEFFIT program suite.

Therefore, ignoring the effect of the non-ideal axial ratio $c / a$ on the anharmonic EXAFS oscillation will lead to nonnegligible errors in the obtained results from the analysis procedures using the FEFF code. The calculated expressions of the first four cumulants, the logarithm of amplitude ratios, and the phase differences taking into account the effect of the non-ideal axial ratio $c / a$ are a necessary addition to modifying the anharmonic EXAFS oscillation of h.c.p. crystals in the $F E F F$ code. The obtained results using the present theoretical model for the temperature and wavenumber dependence of the anharmonic EXAFS oscillation of h.c.p. crystals can be effectively used to complete and modify the calculation toolkit of the FEFF code. These additions can improve the accuracy of the anharmonic EXAFS data analysis of h.c.p. crystals under the effect of the non-ideal axial ratio $c / a$ in the EXAFS data fitting models using the IFEFFIT program suite.

Here, in the h.c.p. structure of $\mathrm{Zn}$, the effect of the non-ideal axial ratio $c / a$ causes a distorted structure. As a result, the first coordination shell is split into two sub-shells, each one composed of six atoms. In this distorted structure, $\mathrm{Zn}-\mathrm{Zn}$ distances for the six bonds in the (aa) plane are $2.6594 \AA$, and $\mathrm{Zn}-\mathrm{Zn}$ distances for the other six bonds distorted along with the direction of the $c$-axis are $2.9069 \AA$ (the distortion between these bond distances is less than $10 \%$ ).

The appropriate approaches must be based on these characteristics to treat the distorted structure of $\mathrm{Zn}$ in investigations on the anharmonic EXAFS spectra. In the usual 
approach, the cumulant expansion can describe deviations from a distorted Gaussian distribution of the RD function by taking into account contributions on both of the $\mathrm{Zn}-\mathrm{Zn}$ paths corresponding to the above two bond distances.

In this work, we used another approach in the present theoretical model to treat the distorted structure of $\mathrm{Zn}$. This approach considers and calculates an effective path of $\mathrm{Zn}-\mathrm{Zn}$ in the cumulant expansion to describe deviations from a distorted Gaussian distribution of the RD function using the AE potential model that takes into account the contribution of both $\mathrm{Zn}-\mathrm{Zn}$ distances for the bonds in the distorted structure of $\mathrm{Zn}$. The obtained results of $\mathrm{Zn}$ in this work have also presented the suitability of the present approach in investigating the effect of the non-ideal axial ratio $c / a$ on anharmonic of h.c.p. crystals. This approach has also been used by Dalba et al. (1995) to effectively treat the local disorder in amorphous germanium with various bond distances using an effective pair potential in the cumulant expansion.

Thus, the above analysis has shown the effective applicability of cumulant expansion with a suitable approach to describing EXAFS oscillations of distorted materials such as $\mathrm{Zn}$ with the non-ideal axial ratio $c / a$ in the h.c.p. structure.

\section{Conclusions}

In this work, we have extended and developed an effective model to calculate and analyze the effect of the non-ideal axial ratio $c / a$ on the temperature and wavenumber dependence of the anharmonic EXAFS oscillation of h.c.p. crystals. The thermal vibrations of the crystal lattice are described as a system consisting of many phonons, each of which corresponds to a wave that has a frequency $\omega(q)$ with a wavenumber $q$ varying in the first Brillouin zone. The AE potential of h.c.p. crystals depending on the non-ideal axial ratio $c / a$ is obtained from the FSNNC approach that can describe the contribution of the nearest-neighbor atoms to the pair interaction potential of backscattering and absorbing atoms. The EACD model has been perfected based on the extended ACD model to calculate the temperature dependence of the anharmonic EXAFS cumulant effectively.

The obtained temperature-dependent expressions using the EACD model of the first four EXAFS cumulants of h.c.p. crystals can satisfy all their fundamental properties. These expressions can describe both the influences of the quantum effects at low temperatures on the zero-point energy and that of the anharmonic effects at high temperatures on the classical limit. The analytical results of the effect of the non-ideal axial ratio $c / a$ have indicated that this effect increases the logarithm of the amplitude ratio and reduces the phase difference of the anharmonic EXAFS oscillation. The magnitude of this effect is significant and is necessarily taken into account in analyzing the anharmonic EXAFS oscillation of h.c.p. crystals, especially with the axial ratio $c / a$ being much different from $(8 / 3)^{1 / 2}$. The analytical results of the influence of the cumulants on the anharmonic EXAFS oscillation have also indicated the role of high-order cumulants. The third cumulant plays a crucial role in the phase shift calculation, and the fourth cumulant plays an important role in the amplitude reduction calculation. These obtained results are essential and effective in analyzing the experimental data of anharmonic EXAFS signals.

The numerical results for $\mathrm{Zn}$ obtained using the present theoretical model are found to be in good agreement with those obtained from experiments and other theoretical methods at various temperatures. This agreement shows the efficiency of the present theoretical model in analyzing the wavenumber and temperature dependence of EXAFS oscillation of h.c.p. crystals under the effect of the non-ideal axial ratio c/a. It can improve the accuracy of the anharmonic EXAFS data analysis of h.c.p. crystals under the effect of the non-ideal axial ratio $c / a$ based on addition and modification for the calculation toolkit of the FEFF code in the EXAFS data fitting models using the IFEFFIT program suite. The present theoretical model can also be applied to treat other distorted crystals that have low symmetry and isotropy with multiple acoustic phonons.

\section{Acknowledgements}

The author would like to acknowledge two anonymous reviewers for their useful comments and suggestions. The author is thankful to Professor Nguyen Van Hung (VNU Hanoi, Vietnam) and Dr Cu Sy Thang (Vietnam Academy of Science and Technology, Vietnam) for providing original EXAFS data of crystalline zinc. The author would also like to thank Professor Fernando Vila and Professor John Tranquada (University of Washington, USA), Professor Paolo Fornasini (University of Trento, Italy), Professor Andrea Sanson (University of Padova, Italy), Professor Takafumi Miyanaga (Hirosaki University, Japan) and Dr Götz Schuck (HelmholtzZentrum Berlin, Germany) for their helpful discussions and comments on the applicability of cumulant expansion approach for crystalline zinc.

\section{References}

Abd el All, N., Thiodjio Sendja, B., Grisenti, R., Rocca, F., Diop, D., Mathon, O., Pascarelli, S. \& Fornasini, P. (2013). J. Synchrotron Rad. 20, 603-613.

Ankudinov, A. L., Ravel, B., Rehr, J. J. \& Conradson, S. D. (1998). Phys. Rev. B, 58, 7565-7576.

Beccara, S. A. \& Fornasini, P. (2008). Phys. Rev. B, 77, 172304.

Beni, G. \& Platzman, P. M. (1976). Phys. Rev. B, 14, 1514-1518.

Bunker, G. (1983). Nucl. Instrum. Methods Phys. Res. 207, 437-444.

Bunker, G. (2010). Introduction to XAFS: A Practical Guide to X-ray Absorption Fine Structure Spectroscopy. Cambridge University Press.

Connétable, D., Huez, J., Andrieu, É. \& Mijoule, C. (2011). J. Phys. Condens. Matter, 23, 405401.

Crozier, E. D., Rehr, J. J. \& Ingalls, R. (1988). X-ray Absorption: Principles, Applications, Techniques of EXAFS, SEXAFS \& $X A N E S, 1$ st ed., ch. 9, edited by D. C. Koningsberger \& R. Prins. New York: Wiley-Interscience.

Dalba, G. \& Fornasini, P. (1997). J. Synchrotron Rad. 4, 243-255.

Dalba, G., Fornasini, P., Grazioli, M. \& Rocca, F. (1995). Phys. Rev. B, 52, 11034-11043.

Dalba, G., Fornasini, P., Grisenti, R., Pasqualini, D., Diop, D. \& Monti, F. (1998). Phys. Rev. B, 58, 4793-4802.

Dalba, G., Fornasini, P., Grisenti, R. \& Purans, J. (1999). J. Synchrotron Rad. 6, 253-254. 
Dalba, G., Fornasini, P. \& Rocca, F. (1993). Phys. Rev. B, 47, 85028514.

Dalba, G., Fornasini, P., Rocca, F. \& Mobilio, F. (1990). Phys. Rev. B, 41, 9668-9675.

Duc, N. B. (2020). Phys. Scr. 95, 075706.

Duc, N. B., Hung, N. V., Khoa, H. D., Vuong, D. Q. \& Tien, T. S. (2018a). Adv. Mater. Sci. Eng. 2018, 3263170.

Duc, N. B., Tho, V. Q., Tien, T. S., Khoa, D. Q. \& Hieu, H. K. (2018b). Radiat. Phys. Chem. 149, 61-64.

Duc, N. B., Tho, V. Q., Van Hung, N., Khoa, D. Q. \& Hieu, H. K. (2017). Vacuum, 145, 272-277.

Eisenberger, P. \& Brown, G. S. (1979). Solid State Commun. 29, 481484.

Feynman, R. P. (1998). Statistical Mechanics: A Set of Lectures, 1st ed. Boca Raton: CRC Press.

Fornasini, P. \& Grisenti, R. (2015). J. Synchrotron Rad. 22, 1242-1257.

Fornasini, P., Grisenti, R., Dapiaggi, M., Agostini, G. \& Miyanaga, T. (2017). J. Chem. Phys. 147, 044503.

Fornasini, P., Monti, F. \& Sanson, A. (2001). J. Synchrotron Rad. 8, $1214-1220$

Frenkel, A. I. \& Rehr, J. J. (1993). Phys. Rev. B, 48, 585-588.

Freund, J., Ingalls, R. \& Crozier, E. D. (1989). Phys. Rev. B, 39, $12537-$ 12547.

Fujikawa, T. \& Miyanaga, T. (1993). J. Phys. Soc. Jpn, 62, 4108-4122. Ghorai, A. (2018). Acta Phys. Pol. A, 134, 549-556.

Girifalco, L. A. \& Weizer, V. G. (1959). Phys. Rev. 114, 687-690.

Greegor, R. B. \& Lytle, F. W. (1979). Phys. Rev. B, 20, 4902-4907.

Grimvall, G. (1999). Thermophysical Properties of Materials, 1st ed. Amsterdam: North-Holland.

Grosso, G. \& Parravicini, G. P. (2000). Solid State Phys. 1st ed., p. 35. London: Academic Press.

Horner, H. (1974). Dynamical Properties of Solids, Vol. 1, ch. 8, edited by G. K. Horton \& A. A. Maradudin. Amsterdam: NorthHolland.

Hung, N. V., Bao Trung, N. \& Kirchner, B. (2010). Physica B, 405, $2519-2525$.

Hung, N. V., Duc, N. B., Vuong, D. Q., Toan, N. C. \& Tien, T. S. (2019). Vacuum, 169, 108872 .

Hung, N. V., Thang, C. S., Duc, N. B., Vuong, D. Q. \& Tien, T. S. (2017a). Eur. Phys. J. B, 90, 256.

Hung, N. V., Thang, C. S., Duc, N. B., Vuong, D. Q. \& Tien, T. S. (2017b). Physica B, 521, 198-203.

Hung, N. V., Tien, T. S., Duc, N. B. \& Vuong, D. Q. (2014). Mod. Phys. Lett. B, 28, 1450174.

Hung, N. V., Tien, T. S., Hung, L. H. \& Frahm, R. R. (2008). Int. J. Mod. Phys. B, 22, 5155-5166.

Jette, E. R. \& Foote, F. (1935). J. Chem. Phys. 3, 605-616.

Jorissen, K. \& Rehr, J. J. (2013). J. Phys. Conf. Ser. 430, 012001.

Kas, J. J., Vila, F. D. \& Rehr, J. J. (2020). International Tables for Crystallogrphy, Volume I, https://doi:10.1107/S1574870720003274.

Kittel, C. (2004). Introduction to Solid State Physics, 8th ed. New York: Wiley.

Lee, P. A., Citrin, P. H., Eisenberger, P. \& Kincaid, B. M. (1981). Rev. Mod. Phys. 53, 769-806.

Lira, I. \& Wöger, W. (2001). Meas. Sci. Technol. 12, 1172-1179.

Lytle, F. W. (1999). J. Synchrotron Rad. 6, 123-134.
Lytle, F. W., Sayers, D. E. \& Stern, E. A. (1975). Phys. Rev. B, 11, 4825-4835.

Mahan, G. D. (1990). Many-Particle Physics, 2nd ed. New York: Plenum.

Marinenko, G. \& Foley, R. T. (1975). J. Res. Natl. Bur. Standards A, 79A, 747-759.

Miyanaga, T. \& Fujikawa, T. (1994). J. Phys. Soc. Jpn, 63, 1036-1052.

Morse, P. M. (1929). Phys. Rev. 34, 57-64.

Negele, J. \& Orland, H. (1972). Quantum Many-Body Systems. New York: Perseus Books.

Newville, M. (2001). J. Synchrotron Rad. 8, 96-100.

Newville, M. \& Ravel, B. (2020). International Tables for Crystallogrphy, Volume I, https:// doi: 10.1107/S1574870720003407.

Podolskaya, E. A. \& Krivtsov, A. M. (2012). Phys. Solid State, 54, 1408-1416.

Ravel, B. \& Newville, M. (2005). J. Synchrotron Rad. 12, 537-541.

Ravel, B. \& Newville, M. (2020). International Tables for Crystallogrphy, Volume I, https://doi.org/10.1107/S1574870720003353.

Rehr, J. J. \& Albers, R. C. (2000). Rev. Mod. Phys. 72, 621-654.

Rehr, J. J., Mustre de Leon, J., Zabinsky, S. I. \& Albers, R. C. (1991). J. Am. Chem. Soc. 113, 5135-5140.

Rennert, P. \& van Hung, N. (1988). Phys. Status Solidi B, 148, 49-61.

Sanson, A. (2010). Phys. Rev. B, 81, 012304.

Sevillano, E., Meuth, H. \& Rehr, J. J. (1979). Phys. Rev. B, 20, $4908-$ 4911.

Stern, E. A., Livnnšs, P. \& Zhang, Z. (1991). Phys. Rev. B, 43, 88508860.

Strauch, D., Pavone, P., Nerb, N., Karch, K., Windl, W., Dalba, G. \& Fornasini, P. (1996). Physica B, 219-220, 436-438.

Tien, T. S. (2020a). J. Theor. Appl. Phys. 14, 295-305.

Tien, T. S. (2020b). J. Phys. D Appl. Phys. 53, 315303.

Tien, T. S. (2021a). Radiat. Phys. Chem. 186, 109504.

Tien, T. S. (2021b). Eur. Phys. J. Plus, 136, 539.

Tien, T. S., Van Hung, N., Tuan, N. T., Van Nam, N., An, N. Q., Minh Thuy, N. T., Kim Lien, V. T. \& Van Nghia, N. (2019). J. Phys. Chem. Solids, 134, 307-312.

Tranquada, J. M. \& Ingalls, R. (1983). Phys. Rev. B, 28, 3520-3528.

Tröger, L., Yokoyama, T., Arvanitis, D., Lederer, T., Tischer, M. \& Baberschke, K. (1994). Phys. Rev. B, 49, 888-903.

Van Hung, N. \& Fornasini, P. (2007). J. Phys. Soc. Jpn, 76, 084601.

Van Hung, N., Hue, T. T., Khoa, H. D. \& Vuong, D. Q. (2016). Physica $B, \mathbf{5 0 3}, 174-178$.

Van Hung, N. \& Rehr, J. J. (1997). Phys. Rev. B, 56, 43-46.

Vérité, G., Willaime, F. \& Fu, C. C. (2007). Solid State Phenom. 129, $75-81$.

Vila, F. D., Spencer, J. W., Kas, J. J., Rehr, J. J. \& Bridges, F. (2018). Front. Chem. 6, 356.

Xia, X., Wang, Z. \& Gao, Y. (2000). Meas. Technol. 11, 430-435.

Yokoyama, T. (1998). Phys. Rev. B, 57, 3423-3432.

Yokoyama, T., Kobayashi, K., Ohta, T. \& Ugawa, A. (1996). Phys. Rev. B, 53, 6111-6122.

Yokoyama, T., Satsukawa, T. \& Ohta, T. (1989). Jpn. J. Appl. Phys. 28, 1905-1908.

Zabinsky, S. I., Rehr, J. J., Ankudinov, A., Albers, R. C. \& Eller, M. J. (1995). Phys. Rev. B, 52, 2995-3009. 